\title{
CSHP Professional Practice Conference 2013: Poster Abstracts / Conférence sur la pratique professionnelle 2013 de la SCPH : Résumés des affiches
}

Sunday, February 3, 2013 • Dimanche 3 février 2013

Facilitated Poster Sessions: Discussion of original research, award-winning projects, and pharmacy practice projects

Séance animée de présentations par affiches : Discussions sur des projets de recherche originale, des projets primés et des projets dans le domaine de la pratique pharmaceutique

Infectious Diseases and Pediatrics

1. Needs Assessment for Antimicrobial Stewardship in Long-Term Care: A Descriptive Study and Survey

2. Evaluation of an Antimicrobial Stewardship Program in a General Medicine Unit and an Intensive Care Unit using Historical and Concurrent Control Groups

3. Urinary Tract Infections: Leading Initiatives in Selecting Empiric Outpatient Treatment (UTILISE)

4. Dextromethorphan in the Treatment and Prevention of Methotrexate Induced Neurotoxicity in Pediatric Patients with Acute Lymphoblastic Leukemia

5. The Safety and Effectiveness of Dexmedetomidine in the Pediatric Intensive Care Unit (SAD-PICU)

Clinical

1. Evaluation of In-Hospital and Post-Discharge Utilization of Preventative Cardiovascular Pharmacotherapy in Patients who have Undergone Coronary Artery Bypass Graft Surgery

2. Efficacy of a Telepharmacist Directed Anticoagulation Management Service

3. Pharmacists Focus on Opioid Management within an Interprofessional Ambulatory Clinic Specializing in Chronic Pain Disorders

4. Assessment of Patient Post-Operative Pain Management Before and After Implementation of a Pain Management Pathway

5. Evaluation of a Discharge Process Implemented on a StrokeMedicine Unit in a Community Teaching Hospital

\section{Safety}

1. Expanding the Role of Medication Reconciliation Teams in Optimizing Pediatric Outcomes

2. Interdisciplinary Medication Safety Initiative to Improve Narcotic Use Practices in a Post Anesthesia Care Unit (PACU)

3. Analysis of Opioid Incidents Requiring Naloxone Administration

4. Development of a Training Program for Hazardous Drugs Handling

5. Multicenter Study of Environmental Contamination with Hazardous Drugs in Hospitals

Professional Practice

1. Enhancing Collaborative Pharmaceutical Care for Chronic Kidney Disease Patients - Survey of Community Pharmacists

2. Exploring Alternative Funding of Rituximab for Rheumatology Clinic Patients: A Pharmacy Led Interprofessional Collaboration Pilot

3. Development of a Pharmacy Department Teaching Guideline in the Era of Expanded Experiential Education

4. What are the Appropriate Selection Criteria for National Hospital Clinical Pharmacy Key Performance Indicators (cpKPI)?

5. Ranking of Healthcare Programs Based on Health Outcome, Health Costs and Safe Delivery of Care in Hospital Pharmacy Practice

\section{Monday, February 4, 2013 • Lundi 4 février 2013}

1. Evaluation of Students' Experience in the "Foundations for Advanced Pharmacy Practice" Rotation

2. Assessment of Implementing Disease State Education Modules and its Effect on Specific Pharmacist Interventions: AIMS Study

3. Comparison of the Consistent and Consult-Based Pharmacy Practice Models in a Heart Failure Clinic

4. Exploration of a Primary Care Pharmacy Specialty Network Listserv Archive Using Content Analysis

5. Implementation of a United States Pharmacopeia (USP) 797 Compliant Central Intravenous Admixture Program

6. Impact of a Pre-Printed Care Order on Influenza and Pneumococcal Vaccination Rates in Older Inpatients in Medicine Units

7. Drug Samples in Outpatient Units: A Constant Problem

8. Impact and Appreciation of Two Methods Aiming at Reducing Hazardous Drug Environmental Contamination: Centralization of Tube Priming in the Pharmacy and Use of a Closed-System Drug Transfer Device

9. Multicenter Study of Environmental Contamination with Hazardous Drugs in 33 Canadian Hospitals

10. Environmental Contamination with Methotrexate in Canadian Retail Pharmacies

11. Exploration des données de doses définies journalières et jours de traitements en pédiatrie - une analyse comparative 2001-2002, 2005-2006 et 2010-2011

12. Mise à jour des soins pharmaceutiques pédiatriques en clinique de VIH-SIDA

\section{Tuesday, February 5, 2013 • Mardi 5 février 2013}

1. Drug Availability in Canada: What Should Hospital Pharmacists Consider?

2. Perception of the Impact of Drug Shortages on Healthcare Professionals and Patients in Canada

3. Implantation d'une nouvelle règle d'utilisation de la vancomycine : une étude pré-post

4. Does Interprofessional Medication Reconciliation from Admission to Discharge Reduce Post-Discharge Patient Emergency Department Visits and Hospital Readmissions?

5. Dissemination of a Pharmacy-Initiated Medication Outcome Monitoring Procedure for Nurses in a Long-Term Care Hospital

6. Pharmacists' Interventions During the Periodic Medication Review Reduce Preventable Adverse Drug Events

7. What are the Appropriate Candidate Clinical Pharmacy Key Performance Indicators (cpKPI) for Hospital Pharmacists?

8. Evaluation of Cancer Treatment Order Entry by a Clinical Support Pharmacy Technician (Oncology) in a Medical Day Unit

9. Evaluation of the Impact of a Pre-Admission Best Possible Medication History on the Admission Medication Reconciliation Rate among Surgical In-Patients

10. Can a Culture of Safety be Enhanced in a Department of Pharmacy?

11. Removal of Concentrated Electrolyte from Patient Care Areas: A Focus on Magnesium Sulfate Injection: Patient Safety and Drug Shortage Implications

12. A Before and After Study of the Implementation of Bedside Medication Storage and Prefilled Narcotics Syringes to a Post Anesthesia Care Unit 


\section{PPC 2013 POSTER ABSTRACTS / RÉSUMÉS DES AFFICHES DE LA CPP 2013}

\section{Wednesday, February 6, 2013• Mercredi 6 février 2013}

1. Facilitating Improved Glycemic Control in the Non-Critically Ill Inpatient Setting

2, Mechanical Mitral Valve Thrombosis with Dabigatran

3. Quetiapine-Associated Serotonin Syndrome: A Case Report

4. Dabigatran Etexilate: A Qualitative Study of Administration, Adherence, Proper Storage and Patient Satisfaction in Ambulatory Patients

5. Evaluation of Antimicrobial Stewardship Program on Leukemia Service Through Prospective Audit and Feedback

6. Determination of Vancomycin Pharmacokinetics in Neonates to Develop Practical Initial Dosing Recommendations

7. Assessing the Adequacy of Documentation in Patients Receiving Antibiotic Therapy

8. A Survey to Evaluate Critical Care (Medical) Trainees' Perception of Antimicrobial Stewardship Programs in Intensive Care Units

9. Empiric Antibiotic Prescribing for Urosepsis in the Emergency Department

\section{CSHP 2015}

CSHP 2015 is a quality program that sets out a vision of pharmacy practice excellence in the year 2015. Through this project, CSHP challenges hospital pharmacists to reach measurable targets for 36 objectives grouped under 6 goals, all aimed toward the effective, scientific, and safe use of medications and meaningful contributions to public health. CSHP 2015 applies to inpatients and outpatients, community and hospital pharmacists, and all practice settings. Posters identified with a "CSHP 2015" logo are those judged by the CSHP 2015 Steering Committee to be particularly relevant to one or more of the 36 objectives.

\begin{abstract}
SCPH 2015
Le projet SCPH 2015 est un programme axé sur la qualité qui propose une vision de l'excellence en pratique pharmaceutique en l'an 2015. Au moyen de ce projet, la SCPH met les pharmaciens d'établissements au défi d'atteindre les cibles mesurables de 36 objectifs répartis entre 6 buts, visant tous l'utilisation efficace, scientifique et sûre des médicaments ainsi que des contributions significatives à la santé publique. Le projet SCPH 2015 s'applique aux patients hospitalisés et externes, aux pharmaciens d'hôpitaux et communautaires, et à tous les milieux de pratique. Les affiches marquées du logo "SCPH 2015 " sont celles que le Comité directeur du projet SCPH 2015 a jugé particulièrement appropriées à l'un ou l'autre des 36 objectifs.
\end{abstract}

The texts of poster abstracts are published exactly as submitted by the authors and have not undergone any copyediting by the Canadian Journal of Hospital Pharmacy. I Le Journal canadien de la pharmacie hospitalière ña pas soumis le texte des résumés des affiches à une révision linguistique et les publie ici tels que remis par les auteurs.

\section{Needs Assessment for Antimicrobial Stewardship in Long-Term Care: A Descriptive Study and Survey \\ Lisa Dong-Ying Wu, Sandra A N Walker, Marion Elligsen, \\ Lesley Palmay, Andrew Simor, Nick Daneman \\ Sunnybrook Health Sciences Centre, Toronto, ON}

Rationale: Recent evidence suggests long-term care (LTC) patients may benefit from antimicrobial stewardship interventions to optimize antibiotic use through standardization of treatments, indications and duration of therapy.

Objectives: The objectives were to assess antibiotic use and need for antimicrobial stewardship services in LTC and to identify LTC health professionals' perspectives regarding antimicrobial stewardship enhancement at their institution.

Study Design and Methods: A retrospective descriptive study of antibiotic use was conducted among elderly patients $(n=336)$ residing in a 17-unit LTC facility at Sunnybrook Health Sciences Centre, Ontario, Canada between April 1,2011 to March 31,2012 using a computerized database. Sunnybrook LTC health professionals $(\mathrm{n}=15$; 9 physicians, 5 pharmacists and 1 nurse) were surveyed with an online Google ${ }^{\mathrm{TM}}$ Docs Form.

Results: There were 358 patient encounters, 835 antibiotic prescriptions, 275 cultures and 170 sensitivities analyzed. The palliative care unit had the highest number of patient encounters $(28.5 \%)$ and the physical support unit had the highest mean antibiotic orders per patient encounter (4.09). Most antibiotic courses (83.7\%) were $<10$ days. Cephalosporins $(30.1 \%)$ and fluoroquinolones $(28.1 \%)$ were the antimicrobials most frequently used. Urine was the most common culture source $(59.3 \%)$ and Escherichia coli (38\%) was the most common bacteria identified in urine. Most apparent urinary tract infections were treated with ciprofloxacin (34\%); yet sensitivity data revealed $32 \%$ of E.coli were ciprofloxacin-resistant in LTC. The online survey had a $60 \%$ response rate $(9 / 15 ; 4$ physicians, 4 pharmacists, and 1 nurse) and found that $33 \%$ of respondents thought current antibiotic use in LTC was sub-optimal and $67 \%$ regarded antimicrobial stewardship service to be important in LTC.

Conclusion: Our study identified that focussed antimicrobial stewardship may be required in our extended care units. Targeted surveillance of all patients with urine cultures may be the antimicrobial stewardship initiative that would have the greatest impact in our LTC units.

\section{Evaluation of an Antimicrobial Stewardship Program in a General Medicine Unit and an Intensive Care Unit using Historical and Concurrent Control Groups}

Lizanne C Béïque ${ }^{1,2}$, Rosemary Zvonar ${ }^{2}$, Gary E Garber ${ }^{l, 2,3}$

${ }^{\prime}$ The Ottawa Hospital, Ottawa, ON

${ }^{2}$ University of Ottawa, Ottawa, $O N$

${ }^{3}$ Ottawa Hospital Research Institute, Ottawa, ON

Rationale: In 2011, an Antimicrobial Stewardship Program (ASP) was introduced on a General Medicine (GM) unit and an Intensive Care Unit (ICU) in a 1000-bed tertiary care hospital. Since acute care beds are distributed over two geographically distinct campuses with some overlap in services, there was a unique opportunity to examine antimicrobial use using the alternate campus as a comparator.

Objectives: To assess the impact of an ASP on a GM unit and in the ICU using both historical and concurrent control groups.

Study Design and Methods: An ASP was introduced and piloted on a GM unit at Campus 1 and an ICU at Campus 2 between October and December 2011. Patients on these two units (intervention groups) receiving antimicrobial agents were assessed by the ASP team using prospective audit and feedback. Drug utilization as defined daily doses 
(DDDs) and costs were compared using two different controls. For each unit the historical control consisted of antimicrobial utilization and cost on the intervention unit for the same time period in the previous year. The concurrent control consisted of the change in antimicrobial utilization and cost between the same two time periods on a similar unit at the alternate campus without ASP intervention (i.e., GM unit at Campus 2 and ICU at Campus 1).

Results:

GM unit: 113 interventions; 91\% acceptance rate

ICU: 164 interventions; 80\% acceptance rate

Metric

Intervention unit

Concurrent control

(Oct-Dec 2011，（Oct-Dec 2011 with ASP) vs. vs. Oct-Dec

historical control 2010, similar (same unit, unit as

Oct-Dec 2010, intervention no ASP) group, different campus, no ASP)

\begin{tabular}{llcc}
\hline General & $\begin{array}{l}\text { DDDs/1000 } \\
\text { patient-days for } \\
\text { all antimicrobial } \\
\text { agents }\end{array}$ & $-25 \%$ & $+7 \%$ \\
& $\begin{array}{l}\text { Total cost for all } \\
\text { antimicrobial } \\
\text { agents }\end{array}$ & $\begin{array}{c}-45 \% \\
(\mathrm{CAN} \$ 13 \mathrm{~K})\end{array}$ & $\begin{array}{c}+40 \% \\
(\mathrm{CAN} \$ 7 \mathrm{~K})\end{array}$ \\
\hline ICU & $\begin{array}{l}\text { DDDs/1000 } \\
\text { patient-days for } \\
\text { all antimicrobial } \\
\text { agents }\end{array}$ & $-13 \%$ & $+1 \%$ \\
& $\begin{array}{l}\text { Total cost for all } \\
\text { antimicrobial } \\
\text { agents }\end{array}$ & $-49 \%$ & $+26 \%$ \\
& $(\mathrm{CAN} \$ 28 \mathrm{~K})$ & $(\mathrm{CAN} \$ 20 \mathrm{~K})$ \\
\hline
\end{tabular}

Conclusion: Compared to the previous year impressive changes were observed in drug utilization and cost with the introduction of an ASP. These were validated by use of a concurrent control unit. Based on these positive results, the antimicrobial stewardship program was broadened.

\section{Urinary Tract Infections: Leading Initiatives in Selecting Empiric Outpatient Treatment (UTILISE) CSHP2915}

Eric Landry, Linda Sulz, Ali Bell, Lane Rathgeber, Heather Balogh Saskatoon Health Region, Regina, SK

Introduction: Overuse of fluoroquinolone (FQ) antibiotics is associated with outbreaks of MRSA and C. difficile-associated diarrhea and increasing resistance to gram-negative organisms. The Regina Qu'Appelle Health Region (RQHR) has seen increasing E. coli resistance to ciprofloxacin over the last decade. The purpose of this study was to evaluate and optimize empiric treatment of Regina General Hospital (RGH) emergency department (ED) outpatients with uncomplicated UTIs, using antimicrobial stewardship principles to align prescribing with local resistance data and best practice.

Methods: An educational strategy, aimed at ED physicians, presented the changes in RQHR antibiotic resistance patterns, principles of antimicrobial stewardship, the drivers of resistance, and a literature review of best practice for outpatient UTIs. An overview of baseline findings from a retrospective chart review, along with the suggested best practice was also presented. A post-intervention audit was conducted in the same manner as the baseline audit for comparison purposes.

Results: Adherence to best practice significantly increased from $40.6 \%$ pre-intervention, to $65.8 \%$ post-intervention $(\mathrm{P}<0.001$; $\mathrm{OR}=2.81$, $95 \%$ CI 1.51-5.25). There was also a significant change in overall antibiotic selection from pre to post-intervention $(\mathrm{P}<0.001$; $\mathrm{OR}=0.25$, $95 \% \mathrm{CI}=0.11-0.58)$. Further statistical analysis suggests this significance was driven by a decrease in ciprofloxacin use from $32.3 \%$ in preintervention to $10.5 \%$ post-intervention. Future interventions may be required to further improve adherence and to determine what effect this may have on reducing resistance rates of $E$. coli to ciprofloxacin.

\section{Goals and Objectives}

1. To raise awareness of the potential antimicrobial stewardship interventions which exist in the treatment of a very common infection, outpatient urinary tract infections.

2. To provide an example of a simply designed, pharmacist lead, interprofessional collaboration on an antimicrobial stewardship initiative in the emergency room setting.

\section{Self-Assessment Questions}

1. What are some of the consequences of fluoroquinolone overuse?

2. Which antimicrobial resistance rates are issues in your health region?

3. Is there opportunity to influence prescribing habits through educational intervention?

Other CSHP 2015 "winning" success stories will also be highlighted at the end of the session.

\section{Dextromethorphan in the Treatment and Prevention of Methotrexate Induced Neurotoxicity in Pediatric Patients with Acute Lymphoblastic Leukemia \\ Keith Miller, Jessica Stovel \\ London Health Sciences Centre, London, ON}

Background and Rationale: Methotrexate (MTX) is an essential component of treatment for acute lymphoblastic leukemia (ALL). Neurotoxicity is a frequent complication of methotrexate therapy that may affect non-compliance and have potential long-term implications. Dextromethorphan (DM), a non-competitive antagonist of the $\mathrm{N}$-methyl-D-aspartate (NMDA) receptor has been used as a neuroprotectant to prevent and treat neurotoxicity without prohibitive toxicity.

Objective: We report a case series examining the use of DM in the treatment and prevention of MTX neurotoxicity. A retrospective chart review of ALL patients known to have experienced neurologic sequellae secondary to methotrexate and/or patients who received DM during the course of treatment was conducted to determine the prescribing patterns of DM.

Results: 8 ALL pediatric patients ( 6 female, mean age 9 years) after receiving IT MTX (mean 8.1 days) experienced neurological adverse events (vomiting (37\%), headache $(87 \%)$, lethargy $(50 \%)$, seizures $(50 \%)$, encephalopathy $(12 \%)$, arthralgia (37\%), hemiparesis $(62 \%)$, ataxia $(12 \%))$. Six of the patients received dextromethorphan treatment at a mean dose of $3.7 \mathrm{mg} / \mathrm{kg} / \mathrm{day}$. All of the patients treated with DM experienced a resolution of symptoms following a mean of 5 days. Six of these patients who received subsequent doses of IT MTX were treated prophylactically with a mean dose of $2.5 \mathrm{mg} / \mathrm{kg}$ divided twice daily, 24 hours prior to administration and for 48 hours post IT MTX administration. Two of the 6 patients who received prophylactic doses experienced recurrent neurotoxic episodes.

Conclusions: These results suggest that dextromethorphan should be considered as an adjunct therapeutic agent to use in patients who experience methotrexate-induced neurotoxicity. Prophylactic use of 
dextromethorphan may prevent the negative neurologic outcomes observed among pediatric patients with leukemia treated with repeated doses of intrathecal MTX. Further prospective research is required to support the use of DM to prevent or treat MTX neurotoxicity.

\section{The Safety and Effectiveness of Dexmedetomidine in the Pediatric Intensive Care Unit (SAD-PICU) \\ Laura Carney, Jennifer Kendrick, Roxane Carr \\ Children's and Women's Health Centre of $B C$, Vancouver, $B C$}

Background: Critically ill children require sedation for comfort and to facilitate interventions. Dexmedetomidine is a newer sedative with little safety data in pediatrics, particularly with durations of therapy greater than 48 hours.

Objective: To quantify the frequency of adverse events and withdrawal syndromes associated with dexmedetomidine and describe its use for continuous sedation in critically ill children.

Methods: A retrospective medical record review of patients who received dexmedetomidine for sedation in the Pediatric Intensive Care Unit. Adverse events were assessed using a Naranjo Score to determine the likelihood of an association with dexmedetomidine.

Results: Included were 144 patients (median age 34 months (range 0 to 17.7 years)) with 153 treatment courses. Mean infusion rate was 0.42 (SD 0.17; range 0.05 to 2) $\mathrm{mcg} / \mathrm{kg} / \mathrm{h}$. Median therapy duration was 20.5 (range 0.75 to 854.75$)$ hours. Hypotension $(\mathrm{N}=81$ (52.9\%)) and bradycardia ( $\mathrm{N}=38(24.8 \%))$ were the most common adverse events, and were "probably" attributable to dexmedetomidine in 17 (11\%) and 9 $(6 \%)$ of treatment courses, respectively. Agitation and hypertension were the most common withdrawal symptoms observed.

Conclusions: Dexmedetomidine is commonly administered for greater than 24 hours in our institution and is generally well tolerated. Patients receiving dexmedetomidine for over 24 hours should be monitored for withdrawal following discontinuation.

Key Words: Dexmedetomidine, Critical Care, Children, Sedation

\section{Evaluation of In-Hospital and Post-Discharge Utilization of Preventative Cardiovascular Pharmacotherapy in Patients who have Undergone Coronary Artery Bypass Graft Surgery}

Arden R Barry', Sheri L Koshman ${ }^{2}$, Colleen M Norris', David B Ross ${ }^{3}$, Glen J Pearson ${ }^{2}$

${ }^{1}$ Mazankowski Alberta Heart Institute, Alberta Health Services, Edmonton, $A B$

${ }^{2}$ Division of Cardiology, University of Alberta, Edmonton, $A B$

${ }^{3}$ Division of Cardiac Surgery, University of Alberta, Edmonton, $A B$

Rationale: Secondary prevention medications, including acetylsalicylic acid (ASA), statins B-blockers and angiotensin-modulating agents (angiotensin-converting enzyme inhibitors or angiotensin receptor blockers), are recommended in patients who have undergone coronary artery bypass graft (CABG) surgery.

Objective: To evaluate the rate of secondary prevention medication utilization from discharge to one-year post-CABG surgery for a cohort of adult patients at the Mazankowski Alberta Heart Institute in Edmonton, Alberta.

Study Design Methods: A retrospective analysis was performed using a clinical patient registry. A randomly selected subset of patients was invited to evaluate medication utilization at one-year post-surgery using community pharmacy records.
Results: The registry identified 1,031 patients. The mean age was 66 years and $80 \%$ were male. The proportion of patients discharged on all four medications post-CABG surgery was $35 \%$. The individual utilization rates for ASA, statins, B-blockers and angiotensin-modulating agents were $96 \%, 94 \%, 92 \%$ and $42 \%$, respectively. Of the patients invited to participate in the one-year evaluation, 151 (39\%) provided consent. The proportion of patients on all four medications at one-year was $48 \%$. Individual utilization rates for ASA, statins, ß-blockers and angiotensin-modulating agents were $95 \%, 84 \%, 84 \%$ and $65 \%$, respectively.

Conclusion: The rate of utilization of four secondary prevention medications was $35 \%$ at discharge and $48 \%$ at one-year post-CABG surgery. These rates were primarily limited by the low use of angiotensinmodulating agents.

\section{Efficacy of a Telepharmacist Directed Anticoagulation Management Service Cody Hotel', Kurt Schroeder ${ }^{1,2}$, Teryl Gosnell', Theresa Crann², Kevin McDonald ${ }^{2}$ \\ ${ }^{I}$ Interlake Regional Health Authority, Selkirk, MB \\ ${ }^{2}$ Northwest Telepharmacy Solutions, Winnipeg, MB}

Rationale: High staff turnover rates in remote communities are common, and can result in suboptimal care for the patients of those areas. These issues become especially important in situations that require close monitoring of therapy. Pharmacist involvement in warfarin dosing and management has been shown to improve patient outcomes through increased \%-time in range (\%-TIR) and decreased bleeding and thrombotic events.

Description and Implementation: A pharmacist directed anticoagulation program was developed by a telepharmacy service to provide stable, accessible care to a remote community. An advanced directive for automatic pharmacist-assisted warfarin dosing was established for patients in the region. Physicians enrolled patients in the program after giving the first warfarin dose and International Normalized Ratio (INR) test. Telepharmacists entered patient data into the DawnAC computer software, and proceeded to dose warfarin based on INR. Two telepharmacists remotely accessed lab data, faxed patient specific dosing letters to corresponding community health centres, and faxed prescriptions to retail pharmacies. Telepharmacists also contacted patients/caregivers at home, providing education, dosing information, and test dates, or to ask questions pertaining to therapy.

Evaluation: From August 1st, 2011 to July 31st, 2012, there were 66 active patients in the program spread out over 6 small communities in the region. In that period, patients had a $\%$-TIR of $67.1 \%$, were above range $10.6 \%$ of the time, and below range $22.3 \%$ of the time. Aside from clinical outcomes, patients experienced improved quality of care as a result of regular follow-up, continuity of care, and increased access to a pharmacist for education. Barriers included language (Cree vs. English), difficulty in accessing INR testing, and on occasion decreased communication with patients who did not own a phone.

Impact/Importance: The telepharmacist directed service was found to be both an effective and practical method of intervention, resulting in improved outcomes for the patients. 


\section{Pharmacists Focus on Opioid Management within an Interprofessional Ambulatory Clinic Specializing in Chronic Pain Disorders}

\section{CSHP2D15}

Karen $\mathrm{Ng}^{l}$, Victoria Su', Laura Murphy ${ }^{2}$

${ }^{\prime}$ Altum Health, University Health Network, Toronto, ON

${ }^{2}$ University Health Network, Leslie Dan Faculty of Pharmacy, University of Toronto, Toronto, $\mathrm{ON}$

Rationale: A hospital ambulatory clinic focusing on functional rehabilitation for injured workers created new clinical pharmacist positions in 2009. This coincided with the publication of recent Canadian guidelines on opioid use in chronic non-cancer pain (CNCP), and was primarily in response to increasing demand from third party payers for medication assessments and opioid management strategies for patients.

Description: Pharmacists complete medication assessments in collaboration with clinic physicians focusing on chronic pain, opioid dependence, and mental health disorders. Formal reports outlining an opioid management plan for implementation by patients' family physicians are completed. Selected patients engage in interprofessional treatment programs which include pharmacist consultations to recommend and monitor opioid rotations, opioid tapering, initiation of alternative pain medications, and management of side effects.

Steps Taken: After an initial pilot with a 0.5 full time equivalent (FTE) pharmacist, pharmacy services expanded to 3 FTE positions. Pharmacists developed standardized processes and documentation templates for medication assessments and consultations, and participated in expansion of services to patients with substance use disorders.

Evaluation: Retrospective chart review of patients who completed assessment and further pharmacist consultations from January 2011 to June 2012. Patients were identified from billing data and clinical reports. Pharmacists completed 433 medication assessments during the study period. Twenty-nine patients assessed had follow-up pharmacist consultations; of these, 21 patients had opioid reduction as a treatment goal. Average daily morphine equivalence (ME) at the time of assessment was $358 \mathrm{mg}$, and patients reduced their ME by an average of $26 \%$.

Importance: Pharmacists focused on opioid management in clinic patients through medication assessment and consultations. This focus addresses an increasing need for collaboration between pharmacists and community physicians. Future growth opportunities include collaboration with clinic anesthesiologists to optimize pain management, and mentorship of community pharmacists to provide standardized opioid assessments and to collaborate with prescribers in their areas.

\section{Assessment of Patient Post-Operative Pain Management Before and After Implementation of a Pain Management Pathway \\ Melanie MacInnis, James Paul, Lori Olivieri \\ Hamilton Health Sciences, Hamilton, ON}

Rationale: A post-operative pain management algorithm (POPMA) was implemented in collaboration with anesthesiologists and gynecologic surgeons. The POPMA provided guidance for therapy selection based on patient characteristics and promoted oral multi-modal analgesia (OMA) over patient controlled analgesia (PCA).

Objectives: To evaluate the impact of a POPMA on pain control and recovery from surgery. The outcomes measured included prescriber's compliance to the pain algorithm; VAS pain scores; nausea and vomiting; pruritus; sedation; bowel recovery; mobilization; and time to discharge.
Study Design and Methods: After local REB approval, we conducted a retrospective review of 120 consecutive patients, 80 from before the POPMA, and 40 following the introduction of the POPMA. We collected pertinent analgesia outcomes, and medications administered from the time of admission to discharge. The two patient groups were analyzed for differences in continuous outcomes with a t-test, and in categorical variables with a chi square analysis.

Results: The primary mode of analgesia was significantly changed from PCA to OMA ( $\mathrm{p}<0.001)$. Pain scores at rest (adjusted difference $0.88, \mathrm{p}=0.004)$ and with activity $(1.21, \mathrm{p}=0.001)$ were higher in the post group. Nausea was not statistically different on post-op days 1 or 2 $(\mathrm{p}=0.122, \mathrm{p}=0.059)$. There was no change in pruritus, sedation or bowel activity. Patients in the post group trended toward earlier mobilization $(\mathrm{p}=0.144)$. Discharge occurred sooner in the post group $(3.41 \mathrm{~d}$ vs $2.97 \mathrm{~d}$; $\mathrm{p}=0.036)$.

Conclusion: The POPMA was successful in minimizing the use of PCA in post-gynecologic surgery patients. A statistically significant increase in post-operative pain was noted between groups; however this increase is not arguably significant clinically. Patients tended to mobilize sooner in the recovery period without PCA. Discharge home was facilitated by OMA and early mobilization. A trend toward an increase in nausea may be due to oral medication administration on an empty stomach.

\section{Evaluation of a Discharge Process Implemented on a Stroke-Medicine Unit in a Community Teaching Hospital}

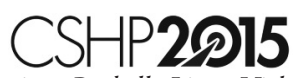

Monica Lee, Parisa Parnian, Rochelle Liem, Vickie Chang, Thomas Chan, Celina Dara, Sharon Eng, Edith Rolko North York General Hospital and Princess Margaret Hospital, University Health Network, Toronto, ON

Rationale: Ensuring the completion of medication reconciliation and patient counselling at the point of discharge may help reduce hospital readmissions. These objectives are also in alignment with the CSHP 2015 goals. We sought to characterize the drug-related issues identified at patient discharge, and the pharmacist workload associated with a newly-implemented discharge process.

Description and Steps Taken: Components of the discharge process were defined. Criteria were developed for identifying patients with complex and/or high-risk medication regimens who would benefit from the discharge process. A database was created to document the issues identified, interventions made and the time spent on each discharge. The process was then implemented on a 35-bed stroke-medicine unit, where patients were previously identified to have a high readmission rate.

Evaluation: During a 6-month period, pharmacists applied the discharge process to $318(77.6 \%)$ of the 410 eligible patients. Discharge medication reconciliation was performed by the physicians in $270(84.9 \%)$ patients. One hundred and ten drug-related issues were noted in 61 (19.2\%) patients. Discrepancies frequently identified were discontinued drug inadvertently resumed upon discharge $(32.7 \%)$, omitted drug (18.2\%), and discrepant dose and/or frequency (8.2\%). Drug therapy problems encountered include inappropriate dose $(9.1 \%)$ and drug not indicated (8.2\%). Discharge counselling was provided to $223(70.1 \%)$ patients and/or their caregivers. Of the patients who did not receive the discharge process, $44.2 \%$ did not have medication reconciliation completed by the physicians. On average, pharmacists spent 25 minutes per discharge process. The mean number of discharge process performed was 2.5 per weekday. 
Importance: Findings indicate that pharmacists play a crucial role in identifying and resolving drug-related issues at patient discharge. The results also imply that adherence to medication reconciliation at discharge may help improve completion of the pharmacist discharge process.

\section{Expanding the Role of Medication Reconciliation Teams in Optimizing Pediatric Outcomes \\ Tassnim Moradipourl, Régis Vaillancourt', Daphne Quiggin', \\ Leandro Avila² \\ ${ }^{I}$ Children's Hospital of Eastern Ontario, Ottawa, ON \\ ${ }^{2}$ University of Waterloo, Waterloo, ON}

Rationale: A standardized Medication Reconciliation (MR) workflow was implemented. The MR process has expanded to include gathering information about the immunization status of patients and the smoking status of patients and parents. The MR process has also been used as a tool to increase the number of flu shots ordered.

Objectives: To describe the MR process and to assess the impact of the expanded role.

Study Design: A retrospective chart audit of patients admitted during the first 7 days of each month for 1 year to assess the key components of the MR program - specifically: medication discrepancies, rates of regular immunizations and flu shots, and smoking status of patients and parents. Results: In the one-year period from October 1, 2011 to September 30, 2012, there were 6069 patients admitted. Of these patients, 68\% (4155) were eligible for MR and of these patients, 92\% (3831) had BPMHs within 24 hours of admission. The retrospective chart audit showed that patients are, on average, on 1.7 medications pre-admission. This totals 1408 medications for the 823 patient charts audited. Of these medications, there were 888 discrepancies of which $27 \%$ (242) were drugs, doses, or instructions that were unintentionally missed or incorrectly ordered on admission. MR identified that $23 \%$ of admitted patients either did not have their regular vaccinations up to date or had unknown vaccination status. MR also identified the $76 \%$ of our patients who did not have their flu shot or had unknown flu vaccination status. Furthermore, we found that nearly $3 \%$ of patients smoked and almost $24 \%$ of patients have a parent that smokes.

Conclusions: CHEO's MR workflow has been effective in identifying medication discrepancies in order to ensure continuation of appropriate medications on admission but its role in patient care may be extended to include increasing the potential for timely immunization and smoking cessation.

\section{Interdisciplinary Medication Safety Initiative to Improve Narcotic Use Practices in a Post Anesthesia Care Unit (PACU)}

\section{CSHP2 15}

Eric Romeril', Melanie MacInnis', Leslie Gauthier ${ }^{l, 2}$, Leslie Gillies', Marianne Kampf', James Paul, ${ }^{l, 2}$

${ }^{1}$ Hamilton Health Sciences, Hamilton, ON

${ }^{2} \mathrm{McM}$ Master University, Hamilton, $O N$

Rationale: The PACU is a high risk environment for medication errors, due to frequency and complexity of medication administration in high acuity patients. Multi-dosing from a single narcotic syringe is prevalent. In addition, a safe work environment for staff members with chemical dependencies needs to be promoted.

Description: A multi-disciplinary committee investigated possible solutions and lead practice change for medication safety in the PACU.
Steps Taken: A focus group analyzed the current medication use process in PACU and developed a process map. This activity identified many high risk practices and environmental factors to which the staff had become tolerant. Solutions were proposed. The chosen solution was manufactured unit dose syringes and bedside lock-boxes. Qualitative data was collected from nurse surveys, observer debrief interviews, and direct observation. Retrospective chart reviews were used prior to the PDSA to select the most frequently administered narcotic doses; and again after the intervention to evaluate the intervention's success.

Evaluation: All nurses at the intervention site reported that they wanted to continue unit dose syringe system. The intervention completely eliminated narcotic waste documentation problems, and showed a trend towards reduction of patient's average pain score. It took 7 hours of Pharmacy technician time a week to manufacture the unit dose syringes. A retrospective chart review uncovered irregularities with medication dispensing and documentation from the automated dispensing cabinet; such that the integrity of signature chain was interrupted. Our team decided to stop using the syringes, as the risk of diversion and medication occurrence was still present and did not offset the resources required to prepare unit dose syringes.

Importance: A multi-disciplinary effort to improve safety of narcotic use in the PACU for both staff and patients was successful in changing nursing practice; however the logistical issues of product production and dispensing limited the success of the project.

\section{Analysis of Opioid Incidents Requiring Naloxone Administration \\ Katherine Lang, Allison Marcil, Lynette Kosar, Zack Dumont, \\ Lisa Ruda, Kaitlyn McMillan \\ Department of Pharmacy Services, Regina Qu'Appelle Health Region (RQHR), Regina, $S K$}

Rationale: Opioid analgesics are high alert medications that are known to cause adverse drug events.

Objective: To determine the cause of opioid incidents that require the administration of naloxone (opioid reversal agent).

Methods: A retrospective chart review of inpatients who received naloxone for reversal of toxicity resulting from licit, in-hospital opioid use was conducted. Cases were analyzed to determine preventability, and preventable cases were assessed to determine the phase of the medication process where incident occurred, as well as the type of incident that occurred (determined through thematic grouping). The drug responsible for toxicity was determined, and the proportion of cases documented by occurrence reporting was noted.

Results: Thirty-six cases were identified, 29 (80.6\%) of which were preventable. The primary medication incident occurred most frequently in the prescribing phase, but multiple phases were often involved. Six types of incidents were identified thematically. Morphine was the drug that most frequently resulted in toxicity. Two $(5.6 \%)$ cases were documented by occurrence reports.

Conclusion: Opioid incidents occurred in the acute care centres under study. Targeted educational initiatives or policy changes are required to decrease the frequency of these incidents and better document their occurrence.

Key Words: medication incident, naloxone, opioid toxicity, adverse drug event 


\section{PPC 2013 POSTER ABSTRACTS / RÉSUMÉS DES AFFICHES DE LA CPP 2013}

\section{Development of a Training Program for Hazardous Drugs Handling}

D M M Woloschuk, W Simoens, L Woods, F Mendelson

Winnipeg Regional Health Authority Pharmacy Program, Winnipeg, MB

Objective: We sought to create an effective, accessible, sustainable, multi-faceted pharmacy staff training program for safe handling of hazardous drugs that could be easily adapted for other disciplines. We also wanted to design a program that would advise staff on an ongoing basis about known or reasonably foreseeable risks to safety and health arising from hazardous drugs used in pharmacy work areas.

Methods: We conducted a needs assessment, then designed and evaluated a set of instructional materials that form a comprehensive training program for safe handling of hazardous drugs. The materials include a slide presentation intended for face-to-face inservices, a self-learning package in hard copy and on-line format, an annual refresher quiz, and a mock spill drill.

Results: Since inception of the training program in 2010, 335 pharmacy staff members have completed one or more training program components. Field tests of each component have improved content, enabled high success rates on refresher quizzes, and identified opportunities to improve the region's Safe Medication Handling policy. The training program has been easy to sustain at a reasonable cost.

Conclusion: Our experience has shown that improving staff safety requires not only a policy and associated procedures, job aides and work tools, but also a comprehensive training program to ensure initial and ongoing use of job aides and work tools by front line staff members. Adoption of the pharmacy hazardous drugs training program for training of nursing personnel region-wide attests to the quality of the program and to pharmacy's medication safety leadership role. Training program materials will be available for viewing during the facilitated poster presentation.

\section{Multicenter Study of Environmental Contamination with Hazardous Drugs in Hospitals}

Jean-François Bussières ${ }^{\prime}$, Cynthia Tanguay ${ }^{I}$, Karine Touzin ${ }^{I}$, Éric Langlois', Michel Lefebure ${ }^{2}$

${ }^{1} \mathrm{CHU}$ Sainte-Justine, Montreal, $Q C$

${ }^{2}$ Centre de toxicologie du Québec (CTQ), Institut National de Santé Publique du Québec (INSPQ), Quebec, QC

Introduction/Objectives: Since the publication of the National Institute for Occupational Safety and Health Alert on hazardous drugs in 2004, many healthcare organizations have reviewed their guidelines and procedures for handling hazardous drugs. Occupational exposure may occur when handling, compounding or administering a drug considered to be hazardous, from storage to waste management. The aim of this project was to measure environmental contamination with cyclophosphamide (CP), ifosfamide (IF) and methotrexate (MTX) in pharmacy and patient care areas of hospitals.

Description of the Project/Methods: Twelve standardized measurement sites within pharmacy (6 sites) and patient care areas (6 sites) were selected. Sites were sampled mid-week at the end of the day. Samples were analyzed for the presence of CP, IF and MTX by UPLC-MS-MS technology. The limit of detection (LOD) was $0.0015 \mathrm{ng} / \mathrm{cm}^{2}$ for CP, $0.0012 \mathrm{ng} / \mathrm{cm}^{2}$ for IF and $0.0060 \mathrm{ng} / \mathrm{cm}^{2}$ for MTX.

Project Experience/Results: A total of 25 hospitals participated in the project (37\% response rate). Overall, 259 samples were collected between April 2008 and January 2010 (147 samples from 25 pharmacy areas and 112 samples from 24 patient care areas). No hospital was using a closed system transfer device (CSTD) at the time of the study. The median[min- max] number of sites per center with at least one positive sample for at least one drug of the three hazardous drugs evaluated was 6[1-12]. A total of $135(52 \%)$ samples were positive for CP, $53(20 \%)$ were positive for IF and $7(3 \%)$ were positive for MTX. The median [min-max] concentration was of $0.0035[<\mathrm{LOD}-28] \mathrm{ng} / \mathrm{cm}^{2}$ for CP, $<\mathrm{LOD}[<\mathrm{LOD}-8.6] \mathrm{ng} / \mathrm{cm}^{2}$ for IF and $<$ LOD $[<L O D-0.58] \mathrm{ng} / \mathrm{cm}^{2}$ for MTX.

Discussion: CP levels were a good indicator to estimate the level of hazardous drug contamination, considering that it is still largely used in most healthcare centers. It also allowed a good comparison with other studies. Our results from 25 hospitals indicated that it is feasible to have a similar (and in some cases, lower) proportion of CP positive surface samples without the use of a closed-system transfer device.

Conclusions: Periodic surface contamination measurements are necessary to ensure that current practices limit healthcare workers occupational exposure to hazardous drugs. This project have helped participating centers identify their specific areas for improvement. The overall results from this project will also serve as an attainable goal that any Canadian hospital may refer to in order to reduce the risks to healthcare workers' safety.

\section{Enhancing Collaborative Pharmaceutical Care for Chronic Kidney Disease Patients - Survey of Community Pharmacists}

Lisa Zhu, Andrea Fox, Yu Chun Chan

Sunnybrook Health Sciences Centre, Toronto, ON

Rational: The Kidney Care Clinic (KCC) at Sunnybrook Health Sciences Centre provides multidisciplinary care for stage 3-5 chronic kidney disease (CKD) patients. As CKD patients are at high risk of drug therapy problems, clinic pharmacists review medications and provide recommendations at each visit. Between clinic visits potential gaps in care exist. Community pharmacists are ideally situated to identify and resolve drug therapy problems arising between visits.

Objectives: The study objectives were to determine: i) community pharmacists' confidence level in managing CKD patients; ii) opportunities for improving collaboration between clinic and community pharmacists; iii) the key clinical information this group would utilize when caring for CKD patients.

Methods: An anonymous survey was sent via mail and online to community pharmacies providing prescription medications to current clinic patients. A total of 318 surveys were sent to 96 pharmacies. Data were analyzed using descriptive statistics including frequencies, ranges and measures of central tendency.

Results: Fifty-one completed surveys were received. Less than a third of pharmacists were aware that a KCC patient was a client of their pharmacy. Ninety percent were confident in providing counseling on medications to manage CKD. Less than two-thirds indicated confidence in recommending drug dosing changes based on kidney function. Over $75 \%$ of pharmacists indicated a willingness to play a greater role in managing CKD patients. All agreed that they would benefit from education on CKD complications and management. Clinical information ranked most useful included an updated medication list with indications and details regarding recent medication changes.

Conclusion: Community pharmacists indicated willingness to have greater involvement in the care of CKD patients. Results reveal a need to increase awareness of clinic patients among providers. Participants were receptive to continuing education and initial efforts should focus on renal drug dosing adjustments and CKD complications. Tools for transferring clinical information require development. 


\author{
Exploring Alternative Funding of Rituximab for \\ Rheumatology Clinic Patients: A Pharmacy Led \\ Interprofessional Collaborative Pilot \\ Bernadette Chevalier', Anne Hiltz', Heather Hemming', \\ Mary Lou Robertson', Alissa Deckerl, Vickie Sullivan', Randi Monroe ${ }^{l}$, \\ Catherine Gaulton', Paula Bond', Donna Gamble', Evelyn Sutton ${ }^{1,2}$, \\ Christy Simpson ${ }^{1,2}$ \\ ${ }^{1}$ Capital Health, Halifax, NS \\ ${ }^{2}$ Dalhousie University, Halifax, NS
}

Rationale and Description of Role: The majority of chronic disease management has shifted from inpatient to outpatient settings without a strategy to ensure our people, facilities and drug resources are appropriately and consistently utilized. To help address this, a pharmacist led interprofessional working group was established and included pharmacy, nursing, social work, medicine, legal, bioethics, and administrative representation. The group drafted a policy to provide an equitable, consistent process in determining how drugs and their administration are funded in outpatient settings. This policy requires patients to use their insurance to pay for their drugs; our institution is the payor of last resort.

Development and Implementation: Rheumatology was selected as a pilot clinic to test and further inform this policy. Rituximab in rheumatoid arthritis patients was targeted as costs had increased from $\$ 96,000$ to $\$ 897,000$ in four years. A Medication Resource Specialist was hired to work with patients and clinic staff around drug insurance reimbursement and pharmaceutical patient assistance program (PPAP) enrollment. Clinic nurses, physicians, and pharmacy staff were inserviced and resource manuals, clinic files, and forms were developed. Information letters were mailed to clinic patients.

Evaluation: Most (90\%) of the 40 patients who received at least one dose during the three month pilot had drug insurance. No clinically significant delays in treatment occurred as a result of investigating insurance. Fifty-one clinic infusions were outsourced and paid for by PPAP. Cost savings for avoided clinic visits based on case costing estimates were $\$ 19,125$. Cost savings for rituximab were $\$ 304,700$. Satisfaction surveys completed by staff, stakeholders, and patients provided valuable feedback.

Future Practice Implications: The pilot demonstrated a successful interprofessional collaboration that resulted in significant cost savings while ensuring high quality patient care. Lessons learned throughout the pilot will be applied to the policy in preparation for its institution-wide roll out.

\section{Development of a Pharmacy Department Teaching Guideline in the Era of Expanded Experiential Education}

Karen Cameron ${ }^{l, 2}$, Cindy Natsheh ${ }^{1,2}$, Emily Musing $g^{1,2}$, Olavo Fernandes ${ }^{1,2}$ ${ }^{1}$ University Health Network, Toronto, ON

${ }^{2}$ Leslie Dan Faculty of Pharmacy, University of Toronto, $O N$

Rationale : The change in academic landscape in pharmacy education has drastically increased the number of student experiential rotations that teaching hospitals need to provide. This has led to a number of issues surrounding pharmacist teaching activities including: 1) consistency in handling requests for teaching, 2) recognition and compensation, and 3) difficulty prioritizing teaching amongst other activities and between the various teaching activities.

Description: In order to address these issues, a consultative process involving front-line pharmacy staff, peer hospitals and academic institutions was used to develop a Pharmacy Department Teaching Guideline. A series of teaching town hall meetings were held inviting participants to provide input on the key issues. A draft of the guideline was developed and shared with staff, peer hospitals and academic institutions for feedback. The guideline was implemented in July 2012.

Evaluation/Results: The resultant operational guideline includes 6 key practical points which deal with: 1) experiential teaching commitments (pharmacist expectations), 2) management of off-site teaching (establishment of pharmacist teaching days), 3) departmental support for preceptor training, 4) consistency in staff recognition, 5) consistency in compensation (honorariums/fees paid to department), and 6) central handling of all teaching requests. After guideline implementation, the number of available departmental student rotations as well as number of pharmacists involved in experiential rotations and off site teaching has increased. We have shared our guideline with peer hospitals who are in varying stages of implementation of a similar model.

Importance: This teaching guideline is applicable and may be of interest to other pharmacy departments facing similar challenges of addressing increased teaching commitments and balancing professional responsibilities.

\section{What are the Appropriate Selection Criteria for National Hospital Clinical Pharmacy Key Performance Indicators (cpKPI)? \\ Natalie Benninger, Richard Slavik, Kent Toombs, Doug Doucette, Jeremy Slobodan, Sean Gorman, Winnie Chan, Bill Semchuk, Cathy Lyder, Olavo Fernandes CSHP National Clinical Pharmacy Key Performance Indicator Task Force, Toronto, ON}

Rationale: A CSHP National Task Force was established to develop a core set of national Clinical Pharmacy Key Performance Indicators (cpKPI) for hospital pharmacists via a systematic (Delphi) evidenceinformed consensus process. Key Performance Indicators (KPIs) are quantifiable measures of quality and an international consensus on appropriate cpKPIs has not been established. The main objectives of this evaluation were to 1) establish a national consensus on cpKPI selection criteria (ideal attributes/properties) 2) construct a Delphi survey instrument and 3) develop consensus evidence tables for key published papers to inform cpKPI selection.

Description: Input was gathered nationally from task force teleconferences as well as national/ local workshops with front-line pharmacy practitioners/leaders. A literature review was conducted and the most important published evidence was identified by cpKPI task force consensus. Systematic evidence tables were created, outlining: the methods/results, strengths/limitations, comparative findings, and proposed highlights for application/synthesis to the cpKPI selection process.

Evaluation: The evidence tables ( $n=7,1$ process and 6 outcome papers) were was used to inform dialogue and debate on a global inventory of candidate cpKPI and to narrow down proposed cpKPI selection criteria. A final set of 11 consensus cpKPI selection criteria ("Slavik-11") were established by utilizing the 5 cpKPI definition characteristics, 4 AHRQ quality indicator parameters and teleconference debate. A Delphi survey instrument, utilizing a 9-point Likert scale, was constructed with the criteria (to be used by the Delphi panel to rank each candidate cpKPI)

Importance: A core set of national cpKPI would serve to harmonize hospital pharmacists toward working together toward optimizing evidenceinformed patient outcomes and permit internal and external benchmarking. A national cpKPI task force established a consensus set of cpKPI selection criteria, a Delphi instrument to rank candidate cpKPI and corresponding evidence tables to support and inform the final determination of a core suite of national cpKPI. 


\section{Ranking of Healthcare Programs Based on Health Outcome, Health Costs and Safe Delivery of Care in Hospital Pharmacy Practice \\ Jean-François Bussières ${ }^{l}$, Lionel Brisseau ${ }^{1}$, Denis Bois ${ }^{2}$, Marc Vallée ${ }^{3}$, \\ Marie-Claude Racine $e^{4}$,André Bonnici \\ ${ }^{1}$ CHU Sainte-Justine, Montréal, QC \\ ${ }^{2}$ Centre hospitalier universitaire de Montréal, Montréal, QC \\ ${ }^{3}$ Centre hospitalier universitaire de Sherbrooke, Sherbrooke, QC \\ ${ }^{4}$ Centre hospitalier universitaire de Québec, Québec, QC \\ ${ }^{5}$ Centre universitaire de santé Mc Gill, Montréal, $Q C$}

Introduction: Given the often limited human and financial resources, managers should consider the best evidence available on a profession's impact to plan healthcare services within an organization. Data are few on ranking healthcare programs in order to prioritize which healthcare program would mostly benefit from the delivery of pharmaceutical care by decentralised pharmacists. The aim of this project was to establish a consensual and coherent ranking of healthcare programs that involve the presence of decentralized pharmacists, based on health outcome, health costs and safe delivery of care.

Project Description: This descriptive study was derived from a structured dialogue (Delphi technique) among directors of pharmacy department. We established a quantitative profile of healthcare programs of five sites that involved the provision of decentralised pharmaceutical care. A summary table of evidence established a unique quality rating per inpatient or outpatient healthcare program. Each director rated the perceived impact of pharmaceutical care per inpatient or outpatient healthcare program on three fields: health outcome, health costs and safe delivery of care. Directors agreed by consensus on the final ranking of healthcare programs.

Project Results: A ranking was assigned for each of the 18 healthcare programs for outpatient care and the 17 healthcare programs for inpatient care involving the presence of pharmacists, based on health outcome, health costs and safe delivery of care. There was a good correlation between ranking based on data from the 2007-2008 Canadian report on hospital pharmacy practice and the ranking proposed by directors of pharmacy department.

Discussion: The use of strength of evidence quality rating combined with Delphi technique allowed our panel experts to propose a final consensual ranking of which healthcare programs would need to benefit from pharmaceutical care. Our study suggested that the ranking by the panellists was influenced by the quality of the evidences available and by current allocation of pharmacists in healthcare programs in Canada.

Conclusions: A novel approach used to rank healthcare programs that include the provision of pharmaceutical care by decentralised pharmacists was described. This ranking approach was based on the perceived impact of pharmaceutical care healthcare program on three fields: health outcome, health costs and safe delivery of care.

\section{Evaluation of Students' Experience in the "Foundations for Advanced Pharmacy Practice" Rotation \\ $V$ Teo, K Lui, A Diamantouros Sunnybrook Health Sciences Centre, Toronto, ON}

Rationale for Report: In September 2011, the Leslie Dan Faculty of Pharmacy began to offer a combined BScPhm - PharmD program providing students in 4th year of the $\mathrm{BScPhm}$ program or those who have completed the BScPhm program, the opportunity to continue toward the completion of a PharmD degree.
Description of Situation: Unlike prior PharmD classes where the prerequisite was at least one year of clinical practice, these students may or may not have had prior clinical experience. The need was identified for a "foundations rotation" in order to ensure a minimum level of preparedness prior to students' advanced clinical rotations. The objective of our project was to gain a deeper understanding of the experiences' of the 7 students involved in the inaugural offering of this rotation at Sunnybrook HSC.

Approach to the Situation: A focus group was conducted with the students on the last day of their rotation. Data collected was transcribed and reviewed by the investigators. Common themes were summarized for discussion.

Evaluation and Results of Project: Students expressed that the foundations rotation improved preparedness for future advanced clinical rotations. Opinions were divergent based on amount of prior clinical experience. Post-baccalaureate students found the rotation to be quite similar to previous structured practical experiential rotations. For students that had no prior experiential experience, they found the rotation overwhelming at times. All students expressed that they were unclear about the objectives of the rotation and the expectations of preceptors.

Usefulness to Future Practice: The experience of these students will be used to ensure improved communication and clarity of expectations on the part of students and preceptors. Further studies are required to determine which specific variables, when modified, will result in the greatest improvement in performance of advanced clinical rotations.

\section{Assessment of Implementing Disease State Education Modules and its Effect on Specific Pharmacist Interventions: AIMS Study \\ Brett Hamilton, Richard Slavik, Victoria Slavik Pharmacy Department - Interior Health Authority, Faculty of Pharmaceutical Sciences - University of British Columbia, Kelowna, BC}

Rationale: Priority disease states are high impact and prevalent diseases that account for a large proportion of health care utilization and costs. Randomized control trials have shown that pharmacists resolving drugrelated problems (DRPs) for patients with priority diseases can improve the overall quality of drug therapy and decrease health care utilization and costs. To promote clinical pharmacist continuous professional development (CPD), eight 4-week disease state education modules (DSEMs) were delivered from January 2009 to December 2011. At the end of each DSEM, a list of key pharmacist interventions (KPI) or evidence-based interventions proven to reduce mortality, morbidity or health care utilization was developed to guide pharmacists' interventions. Current evidence has not proven that pharmacist CPD improves patient care.

Objective: To determine if provision of 4-week DSEMs for clinical pharmacist CPD is associated with objective improvements in patient care. Study Design and Methods: Retrospective, observational study of DRPs resolved by health authority pharmacists from October 1, 2008 to March 31,2012 . The two primary outcomes were the change in proportion of total DRPs that were DSEM DRPs and KPIs from the 6-month PRE module phase compared to the 6-month POST module phase assessed by chi-square analysis with a two-tailed p value less than $0.025(97.5 \%$ confidence interval $[\mathrm{CI}])$.

Results: Primary analysis showed the proportion of total DRPs that were DSEM DRPs increased from $27.9 \%$ to $30.2 \%$; (relative risk increase [RRI], 8.3\%; 97.5\% CI, 1.9-15.3\%). The proportion of total DRPs that were KPIs increased from $21.7 \%$ to $24.4 \%$; (RRI, $12.3 \%$; $97.5 \% \mathrm{CI}$, 4.5-20.8\%). 
Conclusions: There was a statistically significant and clinically important increase in the proportion of DSEM DRPs and KPIs after completion of the DSEMs. This study suggests that using DSEMs for clinical pharmacist CPD on priority disease states are associated with objective improvements in patient care.

\section{Comparison of the Consistent and Consult-Based Pharmacy Practice Models in a Heart Failure Clinic CSHP2D15}

Mei Shi

North York General Hospital, Toronto, ON

Rationale: Although pharmacist interventions in heart failure clinics have demonstrated a reduction in hospitalizations and mortality, the extent of pharmacist involvement and its impact on the type of interventions has not been examined in the literature. The consistent and consult-based pharmacy practice models were compared to evaluate the difference between the types of interventions performed and barriers to providing services.

Description and Steps Taken: One pharmacist assessed patients in both models. In the consistent practice model, the pharmacist assessed all patients scheduled during a full clinic day once weekly. In the consultbased practice model, the pharmacist was contacted at the cardiologist's or nurse's discretion on an ad-hoc basis over the remaining clinic days. Interventions were prospectively documented and categorized as follows: identification of drug therapy problems (DTPs), patient education, therapeutic drug monitoring (TDM), drug information, obtaining a medication history, or a combination of interventions.

Evaluation: Over a 6-month period, the pharmacist assessed 115 patients during 189 encounters in the full-day clinics and 28 patients by consultation. In the consult-based practice model, pharmacist interventions were primarily directed towards performing medication histories $(46.2 \%)$. In the consistent practice model, medication histories represented $2.7 \%$ of the pharmacist's interventions and the remaining time was dedicated to identifying DTPs (46\%), providing education (18\%), a combination of interventions (14.4\%), TDM (14.4\%), and drug information (4.5\%). Barriers to the consult-based practice model included a lack of established relationship with patients, inconsistent follow-up monitoring, and dependency on a physician or nurse for referral.

Importance: Results suggest that a consistent practice model is superior to a consult-based practice model as pharmacists increase their involvement in managing patients' medications to improve safety and outcomes rather than simply performing medication histories. This also aligns with CSHP 2015's objective of managing medication therapy for $70 \%$ of complex patients in ambulatory/specialized care clinics.

\author{
Exploration of a Primary Care Pharmacy Specialty \\ Network Listserv Archive Using Content Analysis \\ Barbara Farrell', Melanie Trinacty ${ }^{2}$, Terri Lisa Sunstrum ${ }^{l}$, \\ Karishma Kak', Terri Schindeb', Lisa Dolovich', Natalie Kennie', \\ Grant Russell', Nancy Waite \\ ${ }^{\prime}$ Bruyère Research Institute, Ottawa, ON \\ ${ }^{2}$ The Ottawa Hospital, Ottawa, ON \\ ${ }^{3}$ Faculty of Pharmacy and Pharmaceutical Sciences, University of Alberta \\ Edmonton, $A B$ \\ ${ }^{4}$ Department of Family Medicine, McMaster University, Hamilton, ON \\ ${ }^{5}$ Summerville Family Health Team, Mississauga, ON \\ ${ }^{6}$ School of Primary Health Care, Monash University, Melbourne, \\ Australia \\ ${ }^{7}$ School of Pharmacy, University of Waterloo, Waterloo, ON
}

Rationale: Canadian pharmacist roles and responsibilities are evolving with increased commitment to primary health care reform and legislation for expanding scope. Little is known about pharmacist educational and support needs in this evolving field. The Canadian Society of Hospital Pharmacists and the Canadian Pharmacists Association jointly host the Primary Care Pharmacist Specialty Network (PC-PSN) which uses a listserv to enable sharing of knowledge.

Objectives: This study examined participant use and educational needs revealed by contributions to the PC-PSN listserv.

Study Design and Methods: Qualitative inductive and deductive content analysis was used to examine one year of archived PC-PSN listserv posts (2010). This was complemented by documenting participation by practice setting, academic affiliation and province. Simple coding using NVivo classified main content of the posts.

Results: One hundred and twenty-nine participants (52.7\% of listserv members) posted to the listserv. Participants worked in family practice $(31 \%)$, community pharmacy $(20 \%)$, hospital pharmacy (12\%), clinics $(11 \%)$ and other $(26 \%)$, with $20 \%$ having an academic affiliation. Over half practiced in Ontario (52.7\%) with others distributed across Canada. Agreement between coders was excellent (0.78); 623 posts were coded. Postings were diverse including patient-specific therapeutic questions, information exchange and practice management needs. Some questions prompted simple answers while others generated conversation and debate. Participants would benefit from education regarding posing comprehensive clinical questions and extrapolating evidence to individual, complex patients. Several participant roles emerged.

Conclusion: The PC-PSN listserv provides participants from varied practice and geographic backgrounds with a forum to discuss issues and solicit input and support. Prominent learning needs that include critical appraisal and formulating focused questions, will assist educators in designing useful education experiences. Participant roles may affect participation and warrant further analysis. Applying this methodology to other listservs would aid understanding of factors that contribute to successful listserv use.

\section{Implementation of a United States Pharmacopeia (USP) 797 Compliant Central Intravenous Admixture Program}

\section{CSHP2D15}

Yuen Chan-Lau, Thomas Chan, Joyce Choy, Christine Ling, Renee Tam, Alefiyah Adamjee, Jocelyn Jackson, Maryam Mohammed Shafiei,

Sarah Truong, Edith Rolko

North York General Hospital, Toronto, ON

Rationale: The objective of implementing a Central Intravenous Admixture (CIVA) program which is compliant with USP 797 standards means Intravenous (IV) medications should be prepared under an International Organization for Standard (ISO) Class 5 environment by trained personnel using aseptic techniques with quality assurance to provide highest quality admixtures.

Description and steps taken: The 4 foci to ensure the CIVA program implementation meet USP 797 standard are: the IV preparation area is constructed to provide an ISO 5 environment, development of policy and procedures for IV admixture personnel training and certification, standardize admixing procedure and worksheets with calculation formulas to ensure admixtures sterility and accuracy, and a vigilant quality assurance process and equipment, an electronic air sampler and incubator, to ensure the sterile preparations meet the USP standards of sterility, stability and expiry.

Evaluation: Prior to CIVA implementation, pharmacy prepared fewer 
than 10 medications in the form of minibag plus and contracted out approximately 5 high usage medications. The implemented program now provides over 50 drugs, including premixed syringes and epidural, minibag plus medications were converted to premixed products. The need for nurses to prepare sterile IV drugs has been reduced by $80 \%$. Drug wastage is monitored and only high usage medications are batched. An average of 20 different admixtures is prepared per day, compared to 20 per month prior to CIVA implementation. The CIVA program is currently staffed with 2 full-time technicians with a daily capacity of over 300 IV bags or syringes.

Importance: The implemented CIVA program ensures IV preparation sterility with an established expiry date and is still growing. Quality assurance monitoring of air and surface in the IV preparation areas and personnel process validation is performed routinely and analyzed. CIVA program implementation enables nurses to have more time to provide other patient care activities.

\section{Impact of a Pre-Printed Care Order on Influenza and Pneumococcal Vaccination Rates in Older Inpatients in Medicine Units \\ Lorie Carter', Sheri Koshman', Margaret Gray ${ }^{3}$, Christine Hughes', Jane Xü, Cheryl Sadowski ${ }^{2}$ \\ ${ }^{1}$ Pharmacy Services, Eastern Health, Marystown, NL \\ ${ }^{2}$ University of Alberta, Edmonton, $A B$ \\ ${ }^{3}$ Pharmacy Services, Alberta Health Services, Edmonton, $A B$}

Rationale: Adults 65 years of age and older are at increased risk of adverse outcomes related to influenza and pneumococcal infections. Vaccinating hospital inpatients is recommended as an important strategy to improve population vaccine coverage, and may be facilitated using a pre-printed care order (PPCO). However, this strategy has received only limited study.

Objectives: To evaluate uptake and impact of a PPCO implemented to improve inpatient influenza and pneumococcal screening and vaccination rates.

Methods: We conducted a retrospective analysis of patients $\geq 65$ years of age admitted to internal medicine at a tertiary care hospital. Charts were randomly selected and reviewed for patients admitted within a three month period during the vaccine campaign in 2010/2011 (pre-PPCO group) and compared to patients admitted during the same three month time period in 2011/2012 (post-PPCO group). Internal medicine pharmacists assisted in the roll-out of the PPCO. Data collection was completed by a single reviewer using standardized forms.

Results: There were 100 patients in the pre group and 77 patients in the post group. Of the examined post group charts, $35 \%$ contained the PPCO, which was fully completed in $65 \%$ of cases. Results were compared using chi-square or fisher's exact tests. Rates of screening were higher in the post group for both influenza vaccination $(75.3 \%$ vs. $13 \%$, $\mathrm{p}<0.0001)$ and pneumococcal vaccination $(80.5 \%$ vs. $11 \%, \mathrm{p}<0.0001)$. Vaccination of eligible patients was also higher in the post group for both influenza vaccination $(33.3 \%$ of 27 patients vs. $6.4 \%$ of 94 patients, $\mathrm{p}<0.001)$ and pneumococcal vaccination $(29.4 \%$ of 34 patients vs. $2.2 \%$ of 92 patients, $\mathrm{p}<0.0001$ ).

Conclusions: Use of a PPCO resulted in higher influenza and pneumococcal screening and vaccination rates in eligible internal medicine inpatients aged $\geq 65$ years. Use of a PPCO with pharmacistsupported roll-out can help contribute to achieving target national immunization rates.

\author{
Drug Samples in Outpatient Units: A Constant \\ Problem \\ Isabelle Barthélémy', Yemsoktheavy Khvan', Hoang Ngan Tina Ly², \\ Suzanne Atkinson', Jean-François Bussières ${ }^{l}$ \\ ${ }^{\prime}$ CHU Sainte-Justine, Montréal, QC \\ ${ }^{2}$ University of Montreal, Montréal, QC
}

Rationale: In Canada, the Food and Drug Act allow the distribution of drug samples to physicians, dentists and pharmacists. Most provincial regulatory authorities do not proscribe their distribution in healthcare settings. Drug sample use may bypass the optimal drug-use process in hospitals and retail pharmacies.

Objectives: The objective of this study is to compare the number of drug samples available in outpatient clinics in a teaching hospital in 2007, 2009 and 2012.

Study Design and Methods: This is a cross-sectional observational study. In our hospital, drug samples were not allowed in patient wards but were tolerated in outpatient clinics. Drug samples were collected by pharmacy staff through unannounced visits. The total number of doses of drug samples was calculated in 2007, 2009 and 2012. A ratio of dose of drug samples per patient visit was also calculated.

Results: A total of number of 78955, 75487 and 91000 doses of drug samples were documented, respectively, in 2007, 2009 and 2012. The ratio of dose of drug samples per patient-visit was respectively of 0.40 , 0.38 and 0.41 . Only $19 \%$ of doses documented were listed on the hospital drug formulary and $4 \%$ of doses were expired. Despite the implementation of a web intranet form to declare drug samples given by industry sales representatives, most drug samples doses were not declared.

Conclusion: The number of drug samples documented in our outpatient clinics has stayed stable for five years. While it appears possible to proscribe their distribution locally in outpatient clinics, it is difficult to do so when regulatory authorities do not proscribe their distribution for a province. We believe drug samples do not contribute to better patient care and should only be dispensed by retail pharmacy through a structured approach with a documentation of doses dispensed in the patient record.

\section{Impact and Appreciation of Two Methods Aiming at Reducing Hazardous Drug Environmental Contamination: Centralization of Tube Priming in the Pharmacy and Use of a Closed-System Drug Transfer Device \\ Annie Guillemette ${ }^{l}$, Hélène Langlois ${ }^{l}$, Maxime Voisine ${ }^{l}$, Delphine Merger ${ }^{2}$, Karine Touzin ${ }^{l}$, Roxane Therrien', Geneviève Mercier', Denis Lebel', Jean-François Bussières ${ }^{1}$ CHU Sainte-Justine (at the time of the study), Montréal, QC ${ }^{2}$ CHU Sainte-Justine, Montréal, QC}

Rationale: The NIOSH alert recommends hazardous drug tubes to be primed in the pharmacy rather than in patient care zones and states that a closed-system drug transfer device (CSTD) may be used when preparing and administering hazardous drugs.

Objectives: To evaluate the impact and appreciation of two methods aiming at reducing hazardous drug environmental contamination: centralization of tube priming in the pharmacy and use of a CSTD.

Study Design and Methods: This is a prospective, experimental and comparative study. Sites in the hematology-oncology satellite pharmacy and care unit were analyzed for the presence of cyclophosphamide, ifosfamide and methotrexate before and after centralization of tube priming in the pharmacy and before and after using a CSTD. The limits of detection for cyclophosphamide, ifosfamide and methotrexate were, 
respectively, of $0.0015 \mathrm{ng} / \mathrm{cm}^{2}, 0.0012 \mathrm{ng} / \mathrm{cm}^{2}$ and $0.0060 \mathrm{ng} / \mathrm{cm}^{2}$. Data was compared with a chi-square test. The pharmacy technician satisfaction was evaluated using a questionnaire.

Results: A total of 225 samples was quantified. After the centralization of tube priming in the pharmacy, no significant difference was found in the proportion of positive samples for cyclophosphamide (15/45(33\%) vs. 10/45(22\%), p=0.239), ifosfamide and methotrexate. Traces of cyclophosphamide found on the floor in patient care areas was significantly reduced (median[min-max] $0.08[0.06-0.09] \mathrm{ng} / \mathrm{cm}^{2}$ vs. $0.03[0.02-0.05], \mathrm{p}<0.0001)$. After using a CSTD, a significant difference was found for the proportion of cyclophosphamide positive samples $(15 / 45(33 \%)$ vs. $0 / 45(0 \%), \mathrm{p}<0.0001)$, but no significant difference was found for ifosfamide $(12 / 45(27 \%)$ vs. 5/45(11\%), $\mathrm{p}=0.059)$ and methotrexate $(1 / 45(2 \%)$ vs. $2 / 45(4 \%), \mathrm{p}=0.557)$. Pharmacy technicians raised issues following the centralization of tube priming (e.g. workload) and the use of CSTD (e.g. spills, particles, workload and handling difficulties).

Conclusion: Centralization of tube priming in the pharmacy did not increase surface contamination in pharmacy, but reduced floor contamination in patient care areas. CSTDs reduced contamination in pharmacy, but issues were raised by pharmacy technicians.

\section{Multicenter Study of Environmental Contamination with Hazardous Drugs in 33 Canadian Hospitals Delphine Merger', Cynthia Tanguay', Éric Langlois', Michel Lefebvre², Jean-François Bussières ${ }^{l}$ \\ ${ }^{I} \mathrm{CHU}$ Sainte-Justine, Montréal, QC \\ Institut national de santé publique du Québec, Québec, QC}

Rationale: A 2004 National Institute for Occupational Safety and Health Alert on hazardous drugs prompted many healthcare organizations to review their guidelines, policies and procedures for the safe use of hazardous drugs. Yet, no safe occupational exposure limit exists.

Objectives: The main objective of this study was to describe environmental contamination with cyclophosphamide, ifosfamide and methotrexate in pharmacy and patient care areas of Canadian hospitals in 2012. The secondary objective was to compare the 2012 environmental monitoring results with the 2008-2010 results.

Study Design and Methods: This is a descriptive and comparative study. Six standardized sites in the pharmacy and six sites on wards were sampled in each participating center. Samples were analyzed for the presence of cyclophosphamide, ifosfamide and methotrexate by UPLCMS-MS. The limit of detection (LOD) was $0.0018 \mathrm{ng} / \mathrm{cm}^{2}$ for cyclophosphamide, $0.0022 \mathrm{ng} / \mathrm{cm}^{2}$ for ifosfamide and $0.008 \mathrm{ng} / \mathrm{cm}^{2}$ for methotrexate. A sample was considered positive if the value was above the LOD. The comparison of surface contamination between the 2008-2010 and 2012 studies was made with the 75th percentile of cyclophosphamide concentration.

Results: A total of 33 hospitals participated in the study and 363 samples were collected. Overall, $40 \%(147 / 363)$ of the samples were positive for cyclophosphamide, $18 \%(68 / 363)$ were positive for ifosfamide and $5 \%(17 / 363)$ were positive for methotrexate. In 2012, the 75 th percentile value of cyclophosphamide surface concentration was of $0.01 \mathrm{ng} / \mathrm{cm}^{2}$, which is four times lower than the 2008-2010 75th percentile of 0.04 $\mathrm{ng} / \mathrm{cm}^{2}$. In both studies, the 75 th percentile for ifosfamide and methotrexate concentration was lower than the LOD.

Conclusion: Surface contamination by hazardous drugs in Canadian hospitals is improving both in terms of the proportions of positive samples and in terms of the surface concentration of hazardous drugs. A local 75 th percentile value should be use to assess local contamination and interpret local results.

\section{Environmental Contamination with Methotrexate in Canadian Retail Pharmacies}

Delphine Merger ${ }^{l}$, Cynthia Tanguay', Éric Langlois', Michel Lefebure², Jean-François Bussières ${ }^{l}$

${ }^{1}$ CHU Sainte-Justine, Montréal, QC

IInstitut national de santé publique du Québec, Québec, QC

Rationale: The NIOSH list of hazardous drugs includes 167 drugs. This growing number of hazardous drugs represents a challenge for retail pharmacies.

Objectives: The objective was to evaluate environmental contamination with methotrexate, cyclophophamide and ifosfamide in Canadian retail pharmacies and to describe practices in Canadian retail pharmacies when handling hazardous drugs.

Study Design and Methods: This is a descriptive and transversal study. Three standardized sites were sampled in each participating retail pharmacy. Samples were analyzed for the presence of cyclophosphamide, ifosfamide and methotrexate by UPLC-MS-MS. The limits of detection (LOD) were $0.10 \mathrm{ng} / \mathrm{mL}, 0.12 \mathrm{ng} / \mathrm{mL}$ and $0.41 \mathrm{ng} / \mathrm{mL}$ for cyclophosphamide, ifosfamide and methotrexate, respectively. A sample was considered positive if the value was above LOD. Nine working practice were assessed in participating retail pharmacies.

Results: Twenty retail pharmacies participated in the study and 60 samples were analyzed. No traces of cyclophosphamide or ifosfamide were detected. Traces of methotrexate were found in 12 out of the 20 pharmacies $(60 \%)$. Twenty-two percent $(13 / 60)$ of the samples were methotrexate-positive: $10 \%(2 / 20)$ from methotrexate containers, $55 \%(11 / 20)$ from pill counter trays used only for hazardous drugs and $0 / 20(0 \%)$ from the preparation counters. Forty percent $(8 / 20)$ of the participating retail pharmacies had a storage space reserved for hazardous drugs and none of them had a preparation area reserved for handling methotrexate tablets. All the participating retail pharmacies had a pill counter reserved for handling hazardous drugs and it was cleaned after use. The nature of the product used to clean the pill counter varied by participant, such as alcohol, soap and water and Wet Ones ${ }^{\circledR}$ wipes. None of the participants cut or crushed methotrexate tablets.

Conclusion: Retail pharmacists must be made aware of the presence of hazardous drugs on working surfaces and of the need to comply with personal protection measures to reduce staff occupational exposure to hazardous drugs.

\section{Exploration des données de doses définies journalières et jours de traitements en pédiatrie - une analyse comparative 2001-2002, 2005-2006 et 2010-2011 \\ Alexia Schott, Justine Guillot, Hélène Roy, Philippe Ovetchkine, Denis Lebel, Jean-François Bussières \\ CHU Sainte-Justine, Montréal, QC}

Justification : L'usage des antibiotiques fait l'objet d'une surveillance accrue. Dans le cadre de nos activités de parrainage des antimicrobiens, nous nous sommes intéressés à la quantification de leur utilisation.

Objectifs : Calculer et discuter les ratios de doses définies journalières (DDJ) et de jours de traitements (JT) par 1000 jours-patients (1000JP) en pédiatrie.

Méthodologie et démarche de l'étude : Une étude rétrospective, transversale, descriptive réalisée au sein d'un centre hospitalier mère-enfant canadien. L'étude porte sur les exercices financiers 20012002, 2005-2006 et 2010-2011 concernant 51 antibiotiques. Sont exclus les antifongiques. Les données ont été extraites du dossier pharmacologique informatisé, couplé aux données relatives aux 
admissions, départs et transferts de patients. Nous avons calculé des ratios de DDJ selon le barème de l'Organisation mondiale de la santé. Bien que les JT soient préférés en pédiatrie, nous avons exploré les deux ratios par $1000 \mathrm{JP}$.

Résultats : Le nombre total de DDJ/1000JP est passé de 402,76 en 2001-2002, à 456,17 en 2005-2006 et 549,56 en 2010-2011. Le nombre total de JT/1000JP est passé de 464,97 en 2000-2001, à 517,44 en 2005-2006 et 657,49 en 2010-2011. Onze antibiotiques ont connu une baisse de leur DDJ/1000JP de 2000-2001 à 2010-2011 [valeur de 2010-2011 représente de 7 à 2000\% de la valeur de 2000-2001] et 9 antibiotiques ont connu une baisse de leur JT/1000 JP de 2000-2001 à 2010-2011 [la valeur de 2010-2011 représente de 7 à 1000\% de la valeur de 2000-2001].

Conclusion : Il existe peu de données quantitatives sur l'utilisation des antibiotiques en pédiatrie. Les programmes de parrainage des antimicrobiens et les autorités gouvernementales exigent des mesures de quantification afin de comparer les profils d'utilisation entre hôpitaux. Il s'agit de la première étude canadienne à décrire sur trois exercices financiers répartis sur 10 années la consommation d'antibiotiques en pédiatrie.

Mise à jour des soins pharmaceutiques pédiatriques en clinique de VIH-SIDA

Freia Stockel, Jean-François Bussières CHU Sainte-Justine, Montréal, QC

Justification : Depuis deux décennies, les pharmaciens hospitaliers exercent majoritairement de façon décentralisée dans les programmes de soins. On reconnait les difficultés inhérentes à la hiérarchisation de ces programmes et des activités pharmaceutiques lorsque les ressources disponibles sont insuffisantes.

Objectif : Mettre à jour le niveau de pratique utilisé en soins pharmaceutiques pédiatriques en clinique de VIH-SIDA.

Méthodologie et démarche : Il s'agit d'une étude descriptive avec revue documentaire menée dans un centre hospitalier universitaire mère-enfant canadien. À partir des meilleures preuves, l'impact du pharmacien en soins VIH-SIDA a été coté selon sept indicateurs de résultats (c.-à-d. mortalité, morbidité, coût, erreurs, effets indésirables, observance et satisfaction). La revue des activités pharmaceutiques en vigueur a servi de base à des discussions sur la mise à jour du secteur.

Résultats : Un total de 52 articles pertinents ont été identifiés et 30 ont été utilisés afin de coter l'impact selon les sept indicateurs. Une revue de l'activité pharmaceutique en 2011-2012, a permis de recenser la réponse à 155 demandes d'information et 199 interventions (43\% ajustement de la pharmacothérapie, $28 \%$ continuité de soins, $16 \%$ bilan comparatif, $13 \%$ autre) pour l'équivalent de 170 heures-travaillées (c.-à-d. couverture clinique de 1-2 jours-semaine) et une cohorte active de près de 70 patients. La mise à jour envisagée du secteur de pratique inclut l'aménagement d'un espace, la documentation écrite des interventions, l'intégration de la fonction à l'équipe de pédiatrie avec jumelage avec un 2ème pharmacien, la rédaction de protocoles et de feuilles d'ordonnances pré-rédigéees, la déclaration proactive d'effets indésirables, la création d'un club de lecture, l'implication dans les écoles, etc.

Conclusion : Il existe peu de données sur la hiérarchisation des programmes de soins et des activités pharmaceutiques. Cette étude décrit une démarche de mise à jour en clinique de VIH-SIDA en pédiatrie.

\section{Drug Availability in Canada: What Should Hospital Pharmacists Consider?}

Andrew Mica, Larry Green

Amgen, Thousand Oaks, CA

Rationale: Drug shortages are an increasing challenge to healthcare in Canada and other countries and can result in the consideration of alternative medications, potentially increasing risk to patients. Although Health Canada maintains a list of drugs in short supply via the Canadian Drug Shortage Database, there is an urgent need for pharmacists to identify potential drug shortages and to understand how manufacturers manage drug supply.

Description: Using biologics as an example, this report highlights supply chain parameters and resources that pharmacists should consider when assessing a manufacturer's ability to maintain and deliver a continuous drug supply.

Steps Taken: As a measure of manufacturer ability to maintain adequate drug supply, inventory turns for 7 major US manufacturers were evaluated between 2006-2010 (low inventory turn denotes a large amount of stock). Key practices employed by leading biologics manufacturers to mitigate the risk of drug shortages were assessed from publicly available data sources, including the US Food and Drug Administration, the American Society of Health System Pharmacists, and manufacturers' websites.

Evaluation: Biotech manufacturers $(\mathrm{n}=2)$ had 1-1.5 turns, large pharmaceutical manufacturers $(\mathrm{n}=2)$ had 1.5-2.0 turns, and generic manufacturers $(n=3)$ had 2-4 turns. Key factors that can enable biologics manufacturers to ensure a continuous supply of products to patients include (1) maintenance of strategic safety stocks, (2) active management of raw material supplies, (3) establishment of redundant manufacturing capabilities and strategic capacity management, (4) active management of robust and secure cold chain distribution networks, and (5) effective integration of global manufacturing systems that link patient demand to production scheduling.

Importance: Understanding and considering how manufacturers manage drug supply should be an integral part of the hospital pharmacist's role when making formulary decisions. Ensuring that approved biologic drugs are available through normal operations and during periods of supply shortages requires leveraging both financial and human resources.

\section{Perception of the Impact of Drug Shortages on Healthcare Professionals and Patients in Canada Isabelle Barthélémy, Denis Lebel, Jean-François Bussières CHU Sainte-Justine, Montréal, QC}

Rationale: In 2011, Sandoz Canada received a warning letter from the Food and Drug Administration. In order to remediate to the nonconformity issues raised, Sandoz suspended and reduced the production of a significant amount of products. That created a major drug shortage crisis in Canadian hospitals.

Objective: To evaluate the perception of the impact of the 2012 drug shortages on healthcare professionals and patients.

Design and Methods: This is an observational study through a web survey in a teaching hospital. A questionnaire of 54 items was developed and pre-tested on five people. A target of 30 responses per health professional category was expected.

Results: A total of 32 physicians, 16 medical residents, 28 pharmacists, 33 nurses and 30 pharmacy technicians participated. $98 \%$ of respondents believed that the current crisis demonstrates the vulnerability of the Canadian market, $79 \%$ have been affected by the crisis and $87 \%$ had to 
modify at least one aspect of their practice. $64 \%$ experienced an increased workload (more than 30 minutes/day for a third of the respondents), $10 \%$ perceived an increase in medication errors, $6 \%$ a prolonged length of stay for some patients and 3\% noted delays for elective surgeries. No respondents could link a death to the drug shortage crisis. $97 \%$ believed the current legal obligations for manufacturers are insufficient and 63\% associated drug shortages to the generic industry. $96 \%$ indicated that there should be at least two Canadian manufacturers for critical drugs. $100 \%$ agreed that drug manufacturer should notify Health Canada before drug shortages or market withdrawal, 63\% mentioned that drug manufacturers should have the obligation to identify a relevant alternative in case of shortage.

Conclusion: These are the first results about healthcare professionals perceptions following the current drug shortages experienced with Sandoz Canada and manufacturers in 2012. Actions from all stakeholders are required.

\section{Implantation d'une nouvelle règle d'utilisation de la vancomycine : une étude pré-post \\ Annie Lavoie, Anais Delicourt, Sophie Penfornis, Denis Lebel, Philippe Ovetchkine, Jean-François Bussières \\ CHU Sainte-Justine, Montréal, QC}

Justification : En 2009, de nouvelles recommandations concernant les modalités d'utilisation de la vancomycine ont été émises. Dans le but de se conformer à ces recommandations, la règle d'utilisation locale a été modifiée.

Objectif : Évaluer la conformité des prescriptions suivant la modification de la règle d'utilisation locale et décrire l'utilisation de la vancomycine dans un hôpital pédiatrique.

Méthodologie : Étude quasi-expérimentale de type pré-post sans groupe témoin. La phase pré s'est déroulée du 01-03-2010 au 30-06-2010 et la phase post du 16-12-2010 et au 02-03-2011. Pour l'intervention, l'écart thérapeutique du prélèvement pré-dose (creux) a été élargi de 5-10 mg/L à $5-15 \mathrm{mg} / \mathrm{L}$ et l'indication pour le prélèvement du post-dose a été précisée. Afin d'améliorer la conformité à la règle, plusieurs actions ont été entreprises : formation d'un comité, approbation par le comité de pharmacologie et diffusion lors de conférences midi. Les posologies et valeurs de prélèvement pré-dose ont été comparées pré et postintervention avec un test $\mathrm{t}$.

Résultats : Des 710 ordonnances de vancomycine rédigées entre le 01-03-2010 et le 02-03-2011, 340 ont été incluses (96 pré et 74 post). Lâge moyen des patients était de $6,8 \pm 5,7$ ans. La posologie initiale quotidienne était de $43 \pm 10,6 \mathrm{mg} / \mathrm{kg} /$ jour pré et de $48,4 \pm 10,8$ $\mathrm{mg} / \mathrm{kg} /$ jour post-intervention $(\mathrm{p}=0,002)$. La valeur moyenne \pm cart-type du prélèvement pré-dose a augmenté de $8,07 \pm 3,8 \mathrm{mg} / \mathrm{L}$ à $10,46 \pm 5,83$ $(\mathrm{p}=0,004)$. La proportion de dosages de type post-dose a diminué de $49 \%$ en pré contre $38 \%$ en post. Le nombre moyen de prélèvements par ordonnance de vancomycine a diminué de 3,1 en pré à 1,5 en post.

Conclusion : Cette étude démontre l'adhésion des prescripteurs à de nouvelles recommandations locales pour l'utilisation de la vancomycine par voie parentérale. Lintervention a permis d'augmenter significativement la dose moyenne initiale et la valeur pré-dose obtenue et de réduire significativement le recours au prélèvement post-dose.

\section{Does Interprofessional Medication Reconciliation from Admission to Discharge Reduce Post-Discharge Patient Emergency Department Visits and Hospital Readmissions?}

Michelle Baker, Chaim M Bell, Wei Xiong, Edward Etchells, Peter Rossos, Kaveh Shojania, Kelly Lane, Tim Tripp, Mary Lam, Kimindra Tiwana, Nita Dhir, Derek Leong, Gary Wong, Jin Huh, Emily Musing, Olavo Fernandes

University Health Network, Toronto, ON

Rationale: Medication reconciliation has been shown to reduce potential adverse drug events but its specific impact on post-discharge hospital readmission is still not known.

Objective: To evaluate the impact of integrated inter-professional (pharmacist-prescriber) medication reconciliation on patient emergency department visits and hospital readmissions.

Study Design and Methods: In this observational cohort study at a tertiary-care hospital, patients discharged by General Internal Medicine (GIM) were identified through administrative databases. The intervention group (patients receiving interprofessional admission to discharge reconciliation supported by an electronic platform) was compared to a control group. The outcome was defined as a composite of emergency department or hospital readmissions within 30 days of the index discharge. A multivariate logistic regression model was used to adjust for age, gender, number of medications, and LACE index.

Results: From 2007-2011, a total of 9,931 unique GIM patient visits ( $\mathrm{n}=8678$ patients) met the criteria of the study. The main analysis did not detect a difference in outcomes between the intervention group $(540 / 2541)$ and control $(1423 / 7390)$ for the primary endpoint. The adjusted odd's ratio was 1.058 (21.25\% vs. $19.26 \%, 95 \%$ CI $0.945-1.19$, $\mathrm{p}=0.326$ ). After propensity score adjustment, the relative risk of readmission was 0.88 ( $16.7 \%$ vs. $18.9 \%$, 95\% CI $0.59-1.32, \mathrm{p}=0.5350)$. Increasing number of medications, LACE index score, as well as male gender were independently correlated with a higher risk of hospital visits. Also, subgroup analyses of high-risk groups: patients $\geq 65$ years, LACE index $\geq 10$, those on high-alert medications, and $\geq 10$ medications did not detect a statistically significant outcome difference between groups.

Conclusion: A 5 year observational evaluation of interprofessional medication reconciliation did not detect a difference in 30 day postdischarge patient hospital visits. Future prospective studies could focus on an enhanced reconciliation intervention bundle on avoidable "medication-related" hospital admissions and post-discharge adverse drug events.

\section{Dissemination of a Pharmacy-Initiated Medication Outcome Monitoring Procedure for Nurses in a Long-Term Care Hospital}

\section{CSHP2D15}

Sophie Pang', Lawrence Jackson ${ }^{2}$, Patricia Gordon ${ }^{3}$

'Pharmacy Student, Leslie Dan Faculty of Pharmacy, Toronto, ON

${ }^{2}$ Department of Pharmacy, Veterans Centre, Sunnybrook Health Sciences Centre, Toronto, $O N$

${ }^{3}$ Department of Nursing, Veterans Centre, Sunnybrook Health Sciences Centre, Toronto, $O N$

Rationale: A Pharmacy-initiated procedure to help nurses monitor and document outcomes from targeted medication interventions had been successfully implemented on two patient care units of a long-term care hospital. The implementation of an electronic documentation system, PointClickCare ${ }^{\circledR}$ (PCC) provided an opportunity to disseminate this 
procedure to the remaining 15 units while orienting all staff to specific features of the PCC system.

Project Description: To disseminate this procedure, a PPC resource guide was developed as an instructional aid. Group and individual nursing in-services were conducted on all units to describe the advantages of this monitoring procedure and provide step-by-step instruction on building a medication Care Plan and creating a medication Alert. Inservices were arranged in collaboration with the PCC Nurse Clinician, and the Advanced Practice Nurse and Patient Care Manager for each unit. A guide for medication monitoring parameters, already in use at the hospital, was employed. Data on the number of medication-related Progress Notes was collected on two units before and after the in-service to assess impact. Nurses' satisfaction with the in-service was obtained using a feedback form and through informal post-session comments.

Evaluation of Project: Nurses valued the medication monitoring guide and found the instructions provided in the in-service easy to understand. The number of medication interventions lacking progress notes fell from $64 \%$ to $48 \%$ and from $43 \%$ to $24 \%$ in the two units respectively, after the in-service. In addition, the number of medication interventions with multiple notes in the subsequent seven days increased slightly.

Importance to Future Practice: Effective dissemination of practice innovations is important for continuous quality improvement. Utilizing a variety of strategies, including in-services, resource guides, one-on-one teaching and audit and feedback, increases the likelihood of success. Insights gained in terms of enablers and barriers to dissemination can be applied to future implementation efforts.

\section{Pharmacists' Interventions During the Periodic Medication Review Reduce Preventable Adverse Drug Events}

\section{CSHP2D15}

Alena Hung ${ }^{1,2}$, Lawrence Jackson ${ }^{2}$, Edward Kung ${ }^{2}$, Victoria Hsu', Dean Yang ${ }^{2}$, Froozan Amin', Evelyn Williams ${ }^{3}$

${ }^{1}$ Doctor of Medicine Candidate, Faculty of Medicine, University of

Manitoba, Winnipeg, $M B$

${ }^{2}$ Department of Pharmacy, Veterans Centre, Sunnybrook Health Sciences Centre, Toronto, $O N$

${ }^{3}$ Department of Medicine, Veterans Centre, Sunnybrook Health Sciences Centre, Toronto, $O N$

Introduction: Preventable adverse drug events (pADEs) are the negative outcomes resulting from suboptimal prescribing, dispensing, administering, or monitoring of a medication. They are common in elderly long-term care patients and can lead to unnecessary harm. The majority of pADEs result from inappropriate prescribing and monitoring. The periodic medication review (PMR) presents an opportunity for pharmacists to identify inappropriate prescribing, intervene, and ultimately reduce the occurrence of preventable adverse drug events.

Objective: To determine the number of therapeutic interventions made by pharmacists at the time of a PMR and their impact on reducing pADEs in a long-term care hospital.

Methods: Data from PMRs conducted in the winter of 2010/2011 was collected retrospectively by reviewing Physician's Orders and pharmacists' notes. The PMR consists of a complete review of each patient's medications by the physician, nurse and pharmacist as well as implementation of solutions to eliminate inappropriate medication use. Any intervention to increase appropriateness was deemed to represent a $\mathrm{pADE}$. To increase the yield of pADEs identified, pharmacists utilized a structured therapeutic thought process based on the Drug Related Problem (DRP) categories of Strand and explicit criteria for prescribing in the elderly (Beers Criteria). Outcomes were compared to data collected in 2004.

Results: Four hundred and fifteen interventions were made in 206 of the 508 residents. Common interventions included: no valid indication (29\%), requires lab monitoring (27\%), dosage or frequency adjustment $(24 \%)$, and new medication required $(10 \%)$. The most frequently involved drug classes were central nervous system (20\%), followed by gastrointestinal $(16 \%)$ and cardiovascular (11\%). Results were comparable to data obtained in 2004 .

Conclusion: Pharmacists, working with physicians and nurses and guided by the patients' goals, can reduce preventable adverse drug events. The PMR serves as an important opportunity for the identification of such pADEs and is an important patient safety procedure.

\section{What are the Appropriate Candidate Clinical Pharmacy Key Performance Indicators (cpKPI) for Hospital Pharmacists?}

\section{CSHP2D15}

Winnie Chan, Doug Doucette, Kent Toombs, Richard Slavik, Jeremy Slobodan, Sean Gorman, Bill Semchuk, Natalie Benninger, Cathy Lyder, Olavo Fernandes

CSHP National Clinical Pharmacy Key Performance Indicator Task Force, Toronto, ON

Rationale: Key Performance Indicators (KPIs) are quantifiable measures of quality. Currently, there is no established national or international consensus on the explicit definition or the core topic foci for clinical pharmacy KPIs (cpKPI). The primary objective of this evaluation was to establish a consensus definition and a core set of Pre-Delphi phase candidate cpKPI via a systematic, national evidenced informed process.

Description: A national cpKPI working group (cpKPIWG), with regional representation, was formed in May 2011 and became a CSHP taskforce in August 2012. Core cpKPI published literature and national (CSHP 2015) measures were reviewed. Feedback was systematically gathered via teleconferences (regional practice model and metric sharing), a national call to members, and national/regional/ local workshops with front-line pharmacy practitioners /leaders on 1) the definition and 2) a potential global inventory of candidate cpKPI.

Evaluation: The consensus definition for a cpKPI included five characteristics. It should 1) reflect a desired quality practice; 2 ) be attributable to direct patient care; 3) be evidence-based to meaningful patient outcomes; 4) be sensitive/suited to pharmacy/pharmacist activities and 5) be feasible to measurable. An "evidence map" summarizing 7 key pharmacy practice papers and other factors was created to help the working group filter and establish 8 final candidate "critical activity/ focused topic "areas ("Doucette-8"). This categorization and a global cpKPI inventory review helped to facilitate debate and discussion in creating a pre-Delphi candidate cpKPI list.

Importance: A national CSHP cpKPI task force established a preDelphi cpKPI consensus definition and an evidence-informed list of candidate cpKPI. These results will be used to inform a national Delphi panel to establish a final core suite national cpKPI will help better define minimum standards and permit benchmark comparisons within and between hospitals. National cpKPI would serve to advance clinical pharmacy practice toward desired quality evidence-informed outcomes. 


\section{Evaluation of Cancer Treatment Order Entry by a Clinical Support Pharmacy Technician (Oncology) in a Medical Day Unit \\ H Neville, L Broadfield, C Harding, S Heukshorst, M Rolle, J Sweetapple \\ Pharmacy Department, Capital Health, Halifax, NS}

Rationale: Pharmacy technicians' responsibilities are expanding to new roles such as the technical task of chemotherapy order entry, which can free pharmacists to provide more clinical services.

Objectives: To determine whether trained clinical support pharmacy technicians (CSPT) enter chemotherapy orders as safely and efficiently as pharmacists. We measured the time needed for order entry, order entry checking, workflow interruptions, and errors with a pharmacist compared to a CSPT.

Study Design and Methods: This was a before and after observational study of oncology order entry for ambulatory hematology patients. Timings were performed by an independent observer using a personal digital assistant. Data were exported to Microsoft Excel for analysis. Difference of means test was used to determine if there was a significant difference between the two phases.

Results: There were 144 and 128 individual orders timed for the pharmacist (Phase 1) and the technician (Phase 2) for order entry, respectively. After outliers were removed, there was no difference in the mean time to enter chemotherapy orders (pharmacist 00:45 sec, technician 00:48 sec, $\mathrm{p}=0.92$ ). There were 114 and 122 individual orders timed for order entry checking by the pharmacist in Phase 1 and Phase 2 , respectively. Phase 2 was significantly faster than Phase 1 (1:12 min vs 1:24 min, $\mathrm{p}<0.05)$. There were 33 non-order entry related interruptions (total 39:38 min) in Phase 1, and 25 interruptions (total 30:08 min) in Phase 2. Errors were 3 in Phase 1 and 0 in Phase 2; all were rated as having no effect on patient care.

Conclusion: Chemotherapy order entry by a pharmacy technician was proven to be not inferior to a pharmacist for safety and efficiency. Changes in scope of practice for clinical support pharmacy technicians can increase the amount of time for direct patient care by pharmacists in the oncology setting.

\section{Evaluation of the Impact of a Pre-Admission Best Possible Medication History on the Admission Medication Reconciliation Rate among Surgical In-Patients}

\section{CSHP2D15}

Tatiana Lee, Thomas Chan, Sharon Eng, Christine Ling, Edith Rolko, Monica Lee

North York General Hospital, Toronto, ON

Rationale: At North York General Hospital, patients scheduled for elective surgeries are required to attend the Pre-Admission Clinic (PAC) for an initial assessment prior to their procedures. This involves obtaining a Best Possible Medication History (BPMH) by the PAC pharmacist, thereby facilitating the completion of medication reconciliation upon the patients' admission to the surgical units. We examined the impact of this process on admission medication reconciliation rates among surgical in-patients.

Description and Steps Taken: To characterize the workflow of the PAC pharmacist and evaluate its impact on admission medication reconciliation rates. Information gathered over a two-week period included: patient characteristics, days elapsed between PAC visit and surgical date, and completion of in-patient BPMH and medication reconciliation. Additionally, differences in BPMH between the PAC encounter and day of surgery were examined.
Evaluation: During the two-week period, $31.8 \%(\mathrm{n}=124)$ of patients assessed at the PAC were admitted after their procedures. Of these, $40.3 \%(n=50)$ have been interviewed by the PAC pharmacist. Patients assessed by the PAC pharmacist were found to have a higher completion rate of admission medication reconciliation $(86.0 \%)$ compared to those who were not $(58.1 \%)$, with a higher proportion completed within the first 24 hours of admission ( $93.0 \%$ vs. $79.1 \%)$. The average number of days between the PAC encounter and surgery was 12.3 , whereby $22.0 \%$ of patients demonstrated differences in their BPMHs. These differences were mostly unintentional discrepancies in documentation $(63.6 \%$, $\mathrm{n}=7$ ), rather than actual changes to medication regimens $(36.4 \%, \mathrm{n}=4)$.

Importance: Findings suggest that a BPMH obtained by a PAC pharmacist facilitates the completion of admission medication reconciliation among surgical in-patients. Intentional changes in medication regimens were uncommon between the time of PAC assessment and day of surgery. Results indicate that all patients who will be admitted post-surgery should have their BPMHs obtained by the PAC pharmacist.

\section{Can a Culture of Safety be Enhanced in a Department of Pharmacy? \\ Heather Kertland, Clarence Chant, Salma Satchu, Jill Garland, \\ Elaine Tom \\ St. Michael's Hospital, Toronto, ON}

Rationale: While pharmacists are key in ensuring the safe use of medications, the culture of safety within the Pharmacy department is not well described in the literature. We sought to assess the departmental safety culture and determine if an intervention that addressed an identified safety concern could improve the overall safety culture.

Methods: An initial departmental safety culture assessment using the validated Hospital Survey on Patient Safety Culture survey was conducted. The findings of the survey were then used to establish the intervention: an open communication forum termed Safety Rounds to discuss medication incidents. These rounds were held twice monthly for four months, followed one month later by a repeat survey.

Results: A total of $71 \%$ of eligible pharmacists completed the baseline survey. The overall safety culture was $46 \%$ positive and the dimensions of concern were: communication openness, feedback and communication about error and hospital handoffs and transitions. The post intervention survey was completed by $50 \%$ of eligible pharmacists. The overall safety culture did not change ( $43 \%$ positive) however there were substantial changes in the proportion of positive responses in several dimensions including: feedback and communication about errors (25 to 58\%), communication openness (38 to $51 \%$ ), organizational learning (56 to $71 \%)$, and teamwork within the unit (71 to $88 \%)$. Dimensions that demonstrated a decrease in the proportion of positive responses were: hospital management support (72 to 56\%), handoffs and transitions (14\% to $5 \%$ ), and teamwork across hospital units ( $45 \%$ to $34 \%$ ).

Conclusion: The open communication forum, Safety Rounds did not change the overall culture of safety but led to improvements in important dimensions and identified areas requiring further work.

\section{Removal of Concentrated Electrolyte from Patient Care Areas: A Focus on Magnesium Sulfate Injection: Patient Safety and Drug Shortage Implications

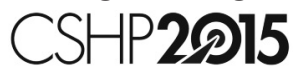

Andrea Beaman

The Credit Valley Hospital and Trillium Health Centre, Mississauga, ON

Rationale: Concentrated magnesium sulfate vials were available on patient care areas for preparation of doses by nurses. The hospital has 
previously removed other concentrated electrolyte solutions from patient care areas. Although magnesium may be administered over 1-2 minutes in patients with persistent pulseless ventricular tachycardia or ventricular fibrillation, there are risks with undiluted administration for non-urgent indications, including hypotension and asystole.

Description of Situation: Removal of concentrated electrolyte solutions from patient care areas is an ISMP initiative and Accreditation Canada Required Organizational Practice to reduce patient risk. Other concentrated electrolytes were removed, but concentrated magnesium remained on many of the patient care areas. Injectable magnesium vials are in short supply nationally, and nurses were using $5 \mathrm{~g}$ single-use vials to prepare $1-4 \mathrm{~g}$ doses, resulting in product waste.

Steps Taken to Implement Change:

- Evaluation of current stock locations and prescribing patterns

- Consultation with Physicians and Clinical Educators in affected patient care areas

- Development of standardized doses and concentrations, and an automatic substitution policy that nurses can apply to STAT doses and pending Pre-Printed Order revisions.

- Education and implementation of switch to magnesium premixed minibags for all patient doses.

- Concentrated magnesium vials retained for crash cart use, and preparation of paediatric doses.

Evaluation: We will benefit from reduced patient-risk; reduced nursing time spent preparing doses, more efficient use of pharmacy services, and reduced waste through implementation of a standardized hospital-wide concentration in premixed magnesium in minibags.

Importance and Usefulness to Practice: This practice change will enhance patient safety and compliance with accreditation requirements. Pharmacists and pharmacy technicians will also function to full scope to ensure patients receive the safest medication options. Stewardship of health care resources through use of auto-sub to reduce wasted nursing time and pharmaceutical resources.

\section{A Before and After Study of the Implementation of Bedside Medication Storage and Prefilled Narcotics Syringes to a Post Anesthesia Care Unit \\ Eric Romeril', Melanie MacInnis' ${ }^{l, 2}$, Leslie Gauthier ${ }^{l, 2}$, Leslie Gillies ${ }^{l}$, \\ Marianne Kampf', James Paul ${ }^{l, 2}$ \\ ${ }^{1}$ Hamilton Health Sciences, Hamilton, ON \\ ${ }^{2} \mathrm{McM}$ Master University, Hamilton, $\mathrm{ON}$}

Rationale: Front line PACU staff asked for help redesigning their medication use system due to safety concerns. It is a high risk environment for medication errors, attributed to patient acuity, frequency of high risk medication administration events (MAE).

Objective: To analyze the impact of introducing bedside-stored medications and prefilled unit-dose syringes on patient, medication errors and nursing practice deviations(NPD).

Methods: This was a REB-approved, blinded, prospective, before-andafter study. The before and after observation periods were each 2 weeks; separated by one week for process change. Two PACUs were chosen from the same multi-site tertiary care hospital system; one served as intervention site and one as control. Trained observers used a standardized case report form to gather data from MAE at both sites using convenience sampling. Data collection and analysis was blinded. Relative risk with associated $95 \%$ confidence interval (CI) is used to report the estimates for binary outcomes, $\mathrm{p}$ values are calculated using Student's $\mathrm{T}$ test.

Results: MAE were recorded at the control (before[n=36], after[n=92] ) and intervention sites (before[ $[\mathrm{n}=62]$, after[ $\mathrm{n}=90]$ ). After exclusion of incomplete or incorrect records $(n=48)$, the remaining data was analyzed $(\mathrm{n}=280)$. There were not enough MAE observations to analyze the medication error data. A significant reduction in NPD was seen in the control $(\mathrm{ARR}=0.307 \mathrm{p}=0.001[95 \% \mathrm{CI}=0.116-0.471])$. No definite change was seen in the intervention $(\mathrm{ARR}=0.031 \mathrm{p}=0.367[95 \% \mathrm{CI}=$ $-0.041-0.124]$ ). Of all MAE observed $78.9 \%$ were narcotic products. All NPDs $(n=4)$ at the intervention site were waste related before, and none were $\operatorname{after}(\mathrm{n}=0)$.

Conclusions: Due to problems with data collection, observer standardization and lower than anticipated MAE; definitive conclusions cannot be reached regarding medication error rate. Nursing practice deviations related to narcotic wasting were reduced. A significant finding may be reached with a higher number of observed $\mathrm{MAE}$ and more rigorous observer training.

\section{Facilitating Improved Glycemic Control in the Non-Critically III Inpatient Setting

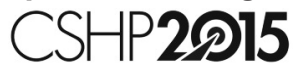

Henry Halapy, Rosemary Tanzini, Catherine Yu St Michael's Hospital, Toronto, ON

Rationale: The use of subcutaneous insulin in the non-critically ill inpatient setting is often characterized by the use of sliding scale insulin in the absence of a scheduled anti-hyperglycemic order, which may result in poor glycemic control.

Description: The purpose of this project was to design and implement preprinted order sets in order to streamline safe, effective insulin use and encourage the use of standing basal-bolus insulin (BBI) and correction factor scales (CF) over sliding scale insulin.

Methods: The preprinted order sets and CF scales were designed and developed by a multi-professional group (diabetes pharmacist, 2 endocrinologists, diabetes nurse educator). They were designed to be easy to use, facilitating ordering of insulin regimens via the use of streamlined check boxes, and encouraged the use of standing BBI regimens coupled with patient-specific standardized CF scales. Order sets were developed for multiple clinical scenarios: initiating multiple dose insulin injections, initiating 1 to 2 insulin injections for type 2 diabetes, maintenance of home insulin regimens, and insulin use in patients who are not eating. The CF scales were incorporated into each individual insulin order set. A hospital wide education program was designed and delivered to clinical pharmacists and nurse educators and physician chiefs to encourage the use of the insulin order sets.

Evaluation: Feedback from clinical pharmacists regarding the clinical use of the order sets was encouraging, but uptake into clinical practice has not been universal throughout the institution. A retrospective chart review comparing pre- and post-implementation performance of the CF scale indicated that the proportion of blood glucose values spent at $>11.0$ $\mathrm{mmol} / \mathrm{L}$ was $39 \%$ lower $(\mathrm{p}=0.03$ ) without an increase in hypoglycemia.

Conclusions: Insulin preprinted order sets have been helpful in encouraging appropriate and safe insulin practice. Challenges remain with increasing universal uptake of the order sets in the institution.

Mechanical Mitral Valve Thrombosis with Dabigatran Jenny Jong, Andrea Narducci, Johanna Proceviat St Michael's Hospital, Toronto, ON

Rationale: While dabigatran etexilate is as effective as warfarin for thromboprophylaxis in atrial fibrillation, the same has not been demonstrated for other indications such as thromboprophylaxis of mechanical heart valves. 
Description: In July 2010, a 26 year-old female underwent a mechanical mitral valve replacement and received warfarin postoperatively. In May 2012 she was switched to dabigatran $150 \mathrm{mg}$ twice daily due to a cutaneous reaction secondary to warfarin. Regular refill history did not suggest adherence to dabigatran was an issue. In August 2012, she presented to hospital with pulmonary edema and cardiogenic shock. A transesophageal echocardiogram revealed a large mobile mass attached to the mitral valve and a mean transmitral gradient of $25 \mathrm{mmHg}$ (normal $<2 \mathrm{mmHg}$ ). During valve replacement surgery, minimal pannus was noted and the existing valve was extensively thrombosed with one leaflet completely fixed and immobile.

Assessment of Causality: The literature indicates prosthetic valve thrombosis (PVT) can occur within a month of inadequate anticoagulation.

Evaluation of the Literature: There are three published case reports that describe both mitral and aortic PVT with dabigatran anticoagulation, all presenting within two months of switching from warfarin. One in vitro study demonstrated that low-dose rivaroxaban resulted in higher thrombus burden on mechanical valves compared to heparin, enoxaparin or high-dose rivaroxaban. A search of clinical trial registries for all of the new oral anticoagulants and prosthetic valves found only RE-ALIGN (NCT 01452347), a phase II study assessing the safety and pharmacokinetics of dabigatran 150, 220 and $300 \mathrm{mg}$ po twice daily.

Importance to Pharmacy Practitioners: Dabigatran and other new oral anticoagulants should not be used as an alternative to warfarin in patients with mechanical valves until efficacy can be established. One hypothesis is that higher doses will need to be used for this indication.

\section{Quetiapine-Associated Serotonin Syndrome: A Case Report \\ Mayce Al-Sukhni $i^{I}$, Mark McIntyre \\ ${ }^{1}$ University of Toronto, Toronto, $\mathrm{ON}$ \\ ${ }^{2}$ Mount Sinai Hospital, Toronto, ON}

Rationale: Quetiapine is an atypical antipsychotic used for treating schizophrenia, bipolar disorder, and major depressive disorder. Atypical antipsychotics, including quetiapine, have been implicated in serotonin syndrome in combination with other serotonergic agents. We describe a novel case of serotonin syndrome possibly due to the combination of risperidone and quetiapine.

Description: A 57-year-old female with a history of psychotic depression was brought to the emergency department after being found at home with confusion, diaphoresis, and rigidity. Medications on admission included risperidone $5 \mathrm{mg}$ daily and quetiapine $25 \mathrm{mg}$ as needed. She reported increased quetiapine ingestion immediately prior to admission, and her medication vial was empty on discovery. On examination, she was slightly febrile (temperature $=37.8^{\circ} \mathrm{C}$ ); hypertensive (blood pressure $=158 / 91 \mathrm{mmHg}$ ); tachycardic (124 beats/minute); and tachypneic (23 respirations/minute). Her creatine phosphokinase was normal (165 IU/L). The patient was diagnosed with serotonin syndrome. Quetiapine and risperidone were held and the patient's symptoms resolved with supportive management. One week after admission, risperidone was reintroduced at a low dose and was tolerated well. Quetiapine was not reinitiated.

Causality Assessment: Excess stimulation of post-synaptic neurons at serotonin receptor subtypes $5-\mathrm{HT}_{1 \mathrm{~A}}$ and $5-\mathrm{HT}_{2 \mathrm{~A}}$ can cause serotonin syndrome. Both quetiapine and risperidone are antagonists at $5-\mathrm{HT}_{2 \mathrm{~A}}$. Additionally, quetiapine is a partial agonist at $5-\mathrm{HT}_{1 \mathrm{~A}}$. In the current case, there is biological plausibility for the combined antagonism of quetiapine and risperidone at $5-\mathrm{HT}_{2 \mathrm{~A}}$ leading to a relative increase in serotonin concentration at the $5-\mathrm{HT}_{1 \mathrm{~A}}$ receptor and, thus, to serotonin syndrome. The Naranjo score of 4 indicates a possible adverse drug reaction.
Literature Evaluation: Several reports have implicated quetiapine in the development of serotonin syndrome. This is the first such case where a full serotonin agonist was not involved.

Importance to Pharmacy Practitioners: This case suggests that quetiapine taken with risperidone may lead to serotonin syndrome. Pharmacists should be aware of this potential adverse effect.

\section{Dabigatran Etexilate: A Qualitative Study of Administration, Adherence, Proper Storage and Patient Satisfaction in Ambulatory Patients \\ Melissa Lo, Paula Brown, Artemis Diamantouros \\ Department of Pharmacy, Sunnybrook Health Sciences Centre, Toronto, $O N$}

Rationale: Dabigatran is a direct thrombin inhibitor recently approved in Canada for the prevention of stroke in patients with atrial fibrillation (AF). As a newly approved drug, there is a paucity of patient experience with dabigatran and much to be learned from "real-world" use.

Objectives: To gain insight into the experiences and challenges of patients prescribed dabigatran.

Study Design and Methods: A qualitative study was conducted using semi-structured interviews of 23 adult patients with $\mathrm{AF}$ using dabigatran. Patients were identified as taking dabigatran either at discharge from hospital or as ambulatory patients filling their prescription at the outpatient pharmacy. The interviews were recorded, transcribed, and coded for themes using grounded theory methodology.

Results: Common themes relating to drug administration included patients reporting difficulty swallowing the capsules and opening the foil-sealed packaging. In patients with poor dexterity potentially harmful measures were taken to overcome this limitation. Few problems were reported regarding adherence to the twice daily medication regimen as most patients used dosettes. Many patients reported storing their medication in an area inappropriate for the strict storage requirements of this drug. Patients overall preferred dabigatran compared to warfarin due to its perceived greater efficacy and lack of INR monitoring and dietary restrictions. However, patients reported concerns regarding cost and lack of a reversal agent for dabigatran.

Conclusions: Patients generally preferred dabigatran over warfarin therapy for AF. However, there appears to be a lack of assessment to ensure patients are appropriate candidates for dabigatran. Upon initial prescribing of dabigatran, pharmacists should assess the patient's ability to open the packaging, swallow the capsules and resources to pay for the medication. Education should be provided regarding proper storage. These issues should be reinforced and reevaluated regularly to ensure dabigatran is being used appropriately and to reduce the risk of adverse effects or drug ineffectiveness.

\section{Evaluation of Antimicrobial Stewardship Program on Leukemia Service Through Prospective Audit and Feedback}

\section{CSHP2915}

Miranda Sol, Lucie Pivnick ${ }^{2}$, Marilyn Steinberg ${ }^{2}$, Tanaz Jivraj', Stephen Lapinsky ${ }^{2}$, Andrew Morris ${ }^{1,2}$, Shahid Husain ${ }^{l}$

${ }^{1}$ University Health Network, Toronto, ON

${ }^{2}$ Mount Sinai Hospital, Toronto, $O N$

Rationale: Patients with haematological malignancies are vulnerable to infections and often receive multiple or prolonged courses of antimicrobials. Emergence of multi-drug resistant organisms and prevalence of C. difficile infections (CDI) have highlighted the need for antimicrobial 


\section{PPC 2013 POSTER ABSTRACTS / RÉSUMÉS DES AFFICHES DE LA CPP 2013}

stewardship programs (ASP), although literature documenting their impact on this immunocompromised population is limited.

Program: University Health Network launched ASP on Leukemia service in February 2010.

Intervention: Prospective audit-and-feedback: an infectious diseases physician and a pharmacist with advanced training review with the leukemia team $3 \mathrm{x} /$ week all patients on at least one antimicrobial, or have microbiology results pending. Suggestions were made regarding diagnostic investigations; antimicrobial selection; duration; dosing; and adverse effects.

Evaluation: The following were compared retrospectively based on pre-ASP (fiscal year 09/10) and post-ASP (fiscal years 10/12 combined) periods by unpaired t-test: monthly antibiotic and antifungal consumption in defined daily dose per 100 patient-days (DDD/100 PD); monthly antibiotic and antifungal costs/100 PD. Incidence of bacteremias, fungemias, CDI and intensive care admission were examined for trend.

Impact: ASP significantly reduced antibiotic consumption and antifungal costs. Antifungal consumption remained stable, attributed to improved consistency in fluconazole prophylaxis, and significant decrease in consumption of costly antifungals to treat fungemias, which decreased significantly by $62 \%$. Coagulase-negative staphylocci (CNST) bacteremia decreased but gram-negative bacteremia increased. A CDI outbreak in Q3/4 of FY 11/12 drove overall increase in CDI post-ASP. Prospective audit and feedback improved certain clinical outcomes but detailed analyses of patient characteristics (e.g. performance score, duration of neutropenia, central line maintenance) are required to understand the rise in ICU admission and gram-negative bacteremias.

Table 1. Comparison of outcome measures in the Pre-ASP and Post-ASP periods

\begin{tabular}{|c|c|c|c|c|c|}
\hline Parameter & & $\begin{array}{l}\text { FY 09/10 } \\
\text { (Pre-ASP) }\end{array}$ & $\begin{array}{l}\text { FY 10/12 } \\
\text { (Post-ASP) }\end{array}$ & $\begin{array}{l}\text { Pre. Vs. } \\
\text { Post-ASP }\end{array}$ & $\begin{array}{c}\text { p-value } \\
(<0.05 \\
\text { denotes } \\
\text { statistical } \\
\text { significance) }\end{array}$ \\
\hline Antibiotics & DDD & $190.65 \pm 13.6$ & $166.09 \pm 36.45$ & $13.19 \% \downarrow$ & 0.032 \\
\hline & Cost $(\$)$ & $\begin{array}{c}6225.39 \pm \\
611.16\end{array}$ & $\begin{array}{c}5723.99 \pm \\
1031.64\end{array}$ & $7.21 \% \downarrow$ & 0.131 \\
\hline Antifungals & DDD & $104.29 \pm 10.63$ & $106.34 \pm 8.97$ & $1.95 \% \uparrow$ & 0.550 \\
\hline & Cost $(\$)$ & $\begin{array}{c}10511.92 \pm \\
2522.61\end{array}$ & $\begin{array}{c}7690.84 \pm \\
2694.44\end{array}$ & $26.8 \% \downarrow$ & 0.005 \\
\hline Fluconazole & DDD & $50.77 \pm 14.92$ & $59.22 \pm 13.06$ & $16.6 \% \uparrow$ & 0.078 \\
\hline Voriconazole & DDD & $20.1 \pm 5.03$ & $16.57 \pm 4.92$ & $17.6 \% \downarrow$ & 0.052 \\
\hline Liposomal amphotericin & DDD & $6.22 \pm 3.23$ & $4.2 \pm 2.80$ & $32.5 \% \downarrow$ & 0.061 \\
\hline Echinocandins & DDD & $13.10 \pm 8.21$ & $10.58 \pm 7.44$ & $19.3 \% \downarrow$ & 0.359 \\
\hline \multicolumn{2}{|c|}{ Positive blood cultures /total sent (\%) } & $\begin{array}{l}202 / 1807 \\
(11.2 \%)\end{array}$ & $\begin{array}{l}520 / 4230 \\
(12.3 \%)\end{array}$ & & \\
\hline \multicolumn{2}{|c|}{$\begin{array}{l}\text { Most common aerobic gram-positive } \\
\text { isolate and incidence } \\
\text { (\#episode per } 100 \mathrm{PD})\end{array}$} & CNST; 0.709 & CNST; 0.581 & & \\
\hline \multicolumn{2}{|c|}{$\begin{array}{l}\text { Incidence of gram-negative bacteremia } \\
\text { (\# episode per } 100 \mathrm{PD} \text { ) }\end{array}$} & 0.274 & 0.417 & & \\
\hline \multicolumn{2}{|c|}{$\begin{array}{l}\text { Incidence of fungemia } \\
\text { (\# episode per } 100 \text { PD) }\end{array}$} & 0.057 & 0.022 & & \\
\hline \multicolumn{2}{|c|}{ CDI incidence (cases per $100 \mathrm{PD}$ ) } & 0.567 & 0.931 & & \\
\hline \multicolumn{2}{|c|}{ ICU admission per 100 PD } & 0.283 & 0.421 & & \\
\hline
\end{tabular}

\section{Determination of Vancomycin Pharmacokinetics in Neonates to Develop Practical Initial Dosing Recommendations \\ Julianne Kim, Sandra A N Walker, Dolores C Iaboni, Scott E Walker, Marion Elligsen, Michael S Dunn, Vanessa Allen, Andrew Simor, Nick Daneman \\ Sunnybrook Health Sciences Centre, Toronto, ON}

Rationale: Variability in neonatal vancomycin pharmacokinetics (PKs) and lack of consensus for optimal troughs in neonatal intensive care units (NICUs) poses challenges to dosing vancomycin in neonates.

Objective: To determine vancomycin PKs in neonates and evaluate dosing regimens to provide practical initial recommendations to target trough concentrations most commonly used in NICUs.

Methods: Fifty neonates who received vancomycin with at least one set of steady-state levels were evaluated retrospectively. Mean PKs were determined using first-order PK equations and univariate linear regression $(\mathrm{p}<0.2)$ followed by multivariate backward linear regression (MVR) $(p<0.05)$ was completed to identify covariates of clearance $(\mathrm{Cl})$ and volume of distribution (Vd). CART was used to identify any breakpoints for significant covariates of $\mathrm{Cl}$ and $\mathrm{Vd}$. Monte Carlo Simulation (MCS) was used to evaluate initial dosing recommendations for target troughs of $15-20 \mathrm{mg} / \mathrm{L}, 5-20 \mathrm{mg} / \mathrm{L}$ and $\leq 20 \mathrm{mg} / \mathrm{L}$.

Results: MVR identified a linear continuous relationship of the significant variables with $\mathrm{Cl}$ and $\mathrm{Vd}$. However, although the regression equations were significant, they are impractical for clinical application. No CART breakpoints for covariates of $\mathrm{Cl}$ or Vd existed. MCS revealed that $\mathrm{mg} / \mathrm{kg}$ dosing was optimal, where $9-12 \mathrm{mg} / \mathrm{kg}$ iv q8h attained the target of $15-20 \mathrm{mg} / \mathrm{L}$ in $15-21 \%$ of patients and continuous infusion with a loading dose of $10 \mathrm{mg} / \mathrm{kg}$ followed by $25-30 \mathrm{mg} / \mathrm{kg} / 24 \mathrm{~h}$ had a probability of target attainment of $28-32 \%$. Initial dosing of $9-15 \mathrm{mg} / \mathrm{kg}$ iv q12h achieved targets of $5-20 \mathrm{mg} / \mathrm{L}$ and $\leq 20 \mathrm{mg} / \mathrm{L}$ in $56-76 \%$ and $92-99 \%$ of patients, respectively.

Conclusions: Initial vancomycin dosing recommendations for neonates were determined. Due to the variability in neonatal vancomycin $\mathrm{PKs}$, monitoring of serum concentrations is recommended when trough concentrations between $15-20 \mathrm{mg} / \mathrm{L}$ or $5-20 \mathrm{mg} / \mathrm{L}$ are desired

\section{Assessing the Adequacy of Documentation in Patients Receiving Antibiotic Therapy \\ Lacey DeVreese, ${ }^{I}$ Rosemary Zvonar', Gary Garberl, ${ }^{l, 3}$ \\ ${ }^{\prime}$ The Ottawa Hospital, Ottawa, ON \\ ${ }^{2}$ University of Ottawa, Ottawa, ON \\ ${ }^{3}$ Ottawa Health Research Institute, Ottawa, ON}

Rationale: Clear and consistent documentation of antibiotic use in medical charts facilitates communication and regular review of therapy. Appropriate documentation related to antibiotic use has been advocated in the medical literature and by various organizations.

Objective: To assess the adequacy of antibiotic documentation prior to the implementation of an antimicrobial stewardship program. 
Study Design and Methods: Three key aspects of antibiotic use were retrospectively evaluated to assess documentation practices for the purpose of the study: 1) the rationale (indication) for the initiation of antibiotic therapy; 2) reassessment of antibiotic therapy; and 3) the intended duration of therapy. Documentation was considered adequate if the rationale, evidence of reassessment, and a plan for duration of therapy were recorded in the medical chart within 24, 96 and 96 hours of initiation of antibiotic therapy, respectively. Each component was assessed individually and collectively as a bundle (i.e., documentation of all three components in each chart). Inclusion of these three aspects on the Pharmacy Clinical Documentation Form and the proportion of patients considered for transition from intravenous to oral therapy were also recorded.

Results: Of the 75 medical charts reviewed, 76\%, 71\% and 31\% met criteria for adequate documentation of the rationale, reassessment of therapy, and a plan for duration of therapy, respectively. All three bundle components were documented in sixteen charts (21\%). Of 47 Pharmacy Documentation Forms available, rationale was indicated in $69 \%$, there was evidence of reassessment in $51 \%$, and a plan for the duration of therapy was documented in $20 \%$. Twenty-three of the 75 (31\%) charts reviewed had evidence that intravenous to oral transitioning was considered.

Conclusions: Key aspects of antimicrobial use are inconsistently documented in medical charts at our institution. Educational efforts and collaboration with an antimicrobial stewardship team may help to ensure a more systematic approach to antimicrobial documentation.

\section{A Survey to Evaluate Critical Care (Medical) Trainees' Perception of Antimicrobial Stewardship Programs in Intensive Care Units \\ Linda Dresser', Marilyn Steinberg ${ }^{2}$, Miranda So ${ }^{l}$, Chaim Bell, \\ Damon Scales ${ }^{3}$, Andrew Morris, \\ ${ }^{1}$ University Health Network, Toronto, ON \\ ${ }^{2}$ Mount Sinai Hospital, Toronto, ON \\ ${ }^{3}$ Sunnybrook Health Sciences Centre, Toronto, ON}

Rationale: Antimicrobial stewardship program (ASP) is an interprofessional collaboration to optimize antimicrobial therapy and minimize adverse events e.g., C. difficile infections and emergence of resistant pathogens in patients. The initiative is usually led by a pharmacist teamed with a physician (both with infectious diseases training/interests), advising the clinical team on investigations, antimicrobial selection, dosing, duration and adverse effects management. Accreditation Canada has mandated that Antimicrobial Stewardship Programs (ASP) be a Required Operating Procedure for all Canadian acute care hospitals in the next accreditation cycle. Currently, ASPs have been implemented in some intensive care units (ICU) in the Greater Toronto Area (GTA). Medical critical care trainees (CCTs) may have varied experiences in ICUs with and without ASP.

Objective: To determine CCTs' perceptions and attitudes towards interprofessional collaboration through ASPs in the ICU.

Methods: All CCTs who have rotated through GTA ICUs between July 2010 and June 2012 were invited to complete an online, anonymous survey assessing their experiences and attitude towards ASP and stewardship team using a 5-point Likert scale: strongly disagree (1); agree (2); neutral (3); agree (4); strongly agree (5).

Results: Response rate to the survey was 32\% (18/57). Forty-four percent of respondents were familiar with the concept of ASP prior to training as a Critical Care Fellow. During their fellowship, 22\% (4/18) rotated through an ICU that did not have an ASP, while 94\% (17/18) rotated through at least one ICU that had an ASP. Half recommended a change regarding frequency of stewardship rounds.
Table 1. Survey items and responses

Survey items

$\%$ Responded

"strongly agree" or

"agree" combined

The ASP functioned as I expected.

$84 \%(16 / 19)$

I feel that stewardship rounds were appropriately

$87 \%(13 / 15)$

focused on patient care.

I found verbal/written feedback to be useful.

I feel that the ASP increased my knowledge of

$100 \%(18 / 18)$

appropriate antimicrobial use in the ICU.

I feel that this ASP model was an efficient use

$89 \%(16 / 18)$

of my time.

I feel that the ASP affected my autonomy in a

$83 \%(15 / 18)$

NEGATIVE manner.

I feel that the patients in the ICU benefited from

having an ASP in place.

I would like to see changes made to how the ASP team interacts with me/my team.

$17 \%(3 / 18)$

$88 \%(15 / 17)$

$50 \%(9 / 18)$

Conclusion: CCTs consider ASP valuable to both patients and clinicians without posing a threat to their autonomy.

\section{Empiric Antibiotic Prescribing for Urosepsis in the Emergency Department \\ Emily Black', Dawn Dalen', Denise Werry², Edith Blondel-Hill, Mike Ertel \\ ${ }^{\prime}$ Qatar University, Doha, Qatar \\ ${ }^{2}$ Kelowna General Hospital, Kelowna, BC}

Rationale: Urinary tract infections, including urosepsis are a common complaint of patients presenting to the emergency department (ED). Despite increased resistance of urinary pathogens to fluoroquinolones, rates of fluoroquinolone prescribing are on the rise resulting in suboptimal empiric antibiotic prescribing.

Objectives: The primary objective of this retrospective review was to describe choice of empiric antibiotic prescribing in patients with urosespis presenting to the ED. An assessment of patient outcomes and evaluation of time to empiric antibiotic administration were secondary objectives of this study.

Study Design and Methods: A retrospective chart review was conducted of patients over the age of 18 presenting to the ED with urosepsis. Optimal choice of empiric antibiotic selection was determined using a predefined algorithm which considered patient specific factors and local patterns of resistance. Results were summarized using descriptive statistics.

Results: We reviewed 50 consecutive charts of patients who presented to the ED with urosepsis. A total of 27 patients, representing $54 \%$ of the study population, received suboptimal empiric antibiotics. The most common reason antibiotics were considered suboptimal was the use of antibiotics with a known local resistance rate of greater than $10 \%$. The most commonly prescribed agent with resistance rates greater than $10 \%$ was ciprofloxacin. Other contributing reasons for suboptimal antibiotic prescribing in our study population included use of empiric agents that were not sensitive to the pathogen isolated, inappropriate empiric dosing, and double coverage of urosepsis with ciprofloxacin and a second antimicrobial agent. Mean time from presentation to antibiotic administration was 295.6 minutes.

Conclusion: Improvement in the empiric choice of antibiotics and time to administration of antibiotics for the management of urosepsis in the ED is needed. Clinical pharmacists can play a key role in ensuring improved patient care by targeting individual patients with urosepsis to promote optimal empiric antibiotic prescribing. 